\title{
Monodromy in the hydrogen atom in crossed fields
}

\author{
R. H. Cushman \\ Mathematics Institute, University of Utrecht, Budapestlaan 6, 3508 TA Utrecht, \\ The Netherlands \\ D. A. Sadovskií \\ Université du Littoral, Citadelle, Boite Postale 5526, 59379 Dunkerque Cedex, \\ France
}

Received May ??, 1999; accepted ??; communicated by J. D. Meiss

\begin{abstract}
We show that the hydrogen atom in orthogonal electric and magnetic fields has a special property of certain integrable classical Hamiltonian systems known as monodromy. The strength of the fields is assumed to be small enough to validate the use of a normal form $\mathcal{H}_{\mathrm{snf}}$ which is obtained from a two step normalization of the original system. We consider the level sets of $\mathcal{H}_{\text {snf }}$ on the second reduced phase space. For an open set of field parameters we show that there is a special dynamically invariant set which is a "doubly pinched 2-torus". This implies that the integrable Hamiltonian $\mathcal{H}_{\text {snf }}$ has monodromy. Manifestation of monodromy in quantum mechanics is also discussed.
\end{abstract}

PACS: 03.20.+i; $32.60+\mathrm{i}$

Keywords: Singular reduction; Monodromy; Energy-momentum map

\section{Introduction}

This paper studies the hydrogen atom in crossed fields. We consider an integrable approximation. We give a detailed analysis of the geometry of this integrable approximation and show that it has a geometric property called

\footnotetext{
1 e-mail: cushman@math.u11.nl

2 e-mail: sadovski@univ-littoral.fr
}

Preprint submitted to Physica D $\quad R C$ and DS, 5/15/99, printed: 26 May 1999 
monodromy. In addition we show that monodromy is visible in the spectrum of the semiclassical quantization of the crossed fields problem.

\subsection{General description}

In 1980 Duistermaat [1] introduced the concept of monodromy in the study of two degree of freedom integrable Hamiltonian systems. Since that time monodromy has been found and analyzed in several integrable systems of classical mechanics [2-11]. Geometrically, monodromy describes the global twisting of a family (bundle) of invariant 2-tori parameterized by (over) a circle of regular values of the energy momentum map of the integrable system. Its presence is signaled by the existence of a singular fiber of the energy momentum map which is topologically a "pinched torus" [12]. Loosely speaking, if an integrable system has monodromy then it is impossible to label the tori in a unique way by values of the actions.

Since invariant tori are at the foundation of semiclassical Einstein-BrillouinKramers (EBK) quantization of integrable systems, monodromy should manifest itself in the corresponding quantum systems [13-15,10,11]. Because monodromy is quite common in classical integrable systems of two degrees of freedom, it should have many important physical implications in quantum mechanics.

In this paper we show that monodromy is present in the hydrogen atom in crossed magnetic and electric fields. To study monodromy, we use a two step normalization procedure to obtain an integrable approximation. The first step, called Keplerian normalization, is well known [16-19]. We use the recent computation in [20] as our starting point. The second normalization was introduced in $[21,22]$ and has not been used before in perturbed hydrogen atom studies. In this paper we focus on this step and detail a simple averaging procedure which gives principal terms necessary for the analysis of monodromy. Higher orders can be obtained by a more elaborate Lie series calculation [23]. Subsequently we analyze the geometry of the integrable system associated to the integrable second normal form $\mathcal{H}_{\mathrm{snf}}$ in order to show that in an explicitly given open subset of relative field strengths the Hamiltonian $\mathcal{H}_{\text {snf }}$ has monodromy [10]. Finally we should observe that the second normal form can be used for a complete qualitative analysis of the crossed fields system in all possible dynamical regimes. A complete analysis [23] reveals that for different relative strengths of the electric and magnetic fields all qualitatively different possible behaviors envisaged in [21] occur.

In the atom in fields problem our paper has many predecessors. In addition

to the already cited work, we should mention the work on problems with axial 
symmetry [24], which implicitly uses the concept of the second reduced phase space. More importantly, we note [25-27] where the concept of a dynamical $\mathbf{S}^{1}$ symmetry (and its corresponding "third" integral) is visibly present and is used in an analysis. We will also compare our results with the early quantum study of Solov'ev [28].

\subsection{More detailed description}

We continue this introduction with a precise description of the crossed fields problem and give an outline of the geometry of this system. At the end we provide an intuitive description of the geometric aspects of monodromy.

\subsubsection{Hydrogen atom in orthogonal external fields}

The Hamiltonian function of the hydrogen atom in the presence of constant orthogonal magnetic and electric fields $[29,30]$ is

$$
H=\frac{P}{2}-\frac{C}{r}+F Q_{2}+\frac{1}{2} G\left(Q_{2} P_{3}-Q_{3} P_{2}\right)+\frac{1}{8} G^{2}\left(Q_{2}^{2}+Q_{3}^{2}\right) .
$$

with subscripts $(1,2,3)$ equal to $(b, e, p)$ of [20]. The direction of the magnetic and electric fields are, respectively, 1 and $2 ; Q$ 's are the coordinates in physical 3 -space, $\boldsymbol{P}$ is the 3 -vector of conjugate momenta, and $r=|Q|$ is the 3 -space length of $\boldsymbol{Q}$. The first two terms in the right hand side of (1) represent the Kepler Hamiltonian, the third is the electrostatic potential describing Stark effect, and the two last terms describe the linear and quadratic Zeeman effect. We introduce an "effective charge" $C$ in order to have the same kind of parameters as in (1.1) of [21]. The Hamiltonian in [21] is equivalent to (1) with the quadratic Zeeman term omitted and thus can be interpreted as a case when the magnetic field strength $G$ is small.

For $F \neq 0$ and $G \neq 0$ the Hamiltonian function $H(1)$ has no strict continuous symmetry. However, it does have a discrete $\mathbf{Z}_{2} \times \mathbf{Z}_{2}$ symmetry which will be taken into account in our analysis. More information on the symmetry analysis can be found in $[20,23]$.

\subsubsection{Scheme of the analysis and geometry}

To determine whether our Hamiltonian system has monodromy, we normalize $H$ (1) twice and study the resulting integrable system. First we normalize $H$ with respect to the Keplerian symmetry. Truncating at order 2 gives the first normal form $\mathcal{H}_{\text {fnf }}$ which has the regularized Kepler Hamiltonian $H_{0}=2 \mathrm{~N}$ as an 
integral of motion. (For our original Hamiltonian system $2 N$ is an approximate integral of motion). Removing the Keplerian symmetry from the first normal form gives a two degree of freedom Hamiltonian system on $\mathrm{S}_{n / 2}^{2} \times \mathrm{S}_{n / 2}^{2}$, the product of two 2 -spheres of radius $n / 2$. Here $n$ is the value of the Keplerian integral $N$. The coordinates used to describe the first reduced phase space $S_{n / 2} \times S_{n / 2}$ are the Hamiltonian functions corresponding to the vector fields generating the $\mathrm{SO}(4)$ symmetry of $H_{0}$.

To perform the second normalization we look at the first order term $\mathcal{H}_{1}$ in $\mathcal{H}_{\text {fnf }}$. Using the Poisson structure of the $S_{n / 2}^{2} \times S_{n / 2}^{2}$ coordinate functions, we obtain a Hamiltonian vector field $X_{\mathcal{H}_{1}}$ on $\mathrm{S}_{n / 2}^{2} \times \mathrm{S}_{n / 2}^{2}$ whose flow generates an $\mathbf{S}^{1}$ symmetry. This is an approximate symmetry of the first normal form. Averaging $\mathcal{H}_{\text {fnf }}$ with respect to the flow of $X_{\mathcal{H}_{1}}$ gives the second normal form $\mathcal{H}_{\text {snf }}$. Note that $\mathcal{H}_{1}$ is an integral of the Hamiltonian system corresponding to the second normal form. Because $\mathbf{S}^{1}$ action on $M_{c}=\mathcal{H}_{\text {fnf }}^{-1}(c) \cap\left(\mathrm{S}_{n / 2}^{2} \times \mathrm{S}_{n / 2}^{2}\right)$ defined by the flow of $X_{\mathcal{H}_{1}}$ has fixed points when $c=0$, we must use singular reduction [2] to obtain the second reduced phase space $P_{n, c}$. We then investigate the geometry of the level sets of the energy momentum mapping

$$
\mathcal{E} \mathcal{M}: \mathrm{S}_{n / 2}^{2} \times \mathrm{S}_{n / 2}^{2} \rightarrow \mathbf{R}^{2}: p \rightarrow\left(\mathcal{H}_{\mathrm{snf}}(p), \mathcal{H}_{1}(p)\right)
$$

by reducing the $\mathbf{S}^{1}$ symmetry to obtain a one degree of freedom system on $P_{n, c}$. We show that for an explicitly given open set of field parameters the $(0,0)$-level set of $\mathcal{E} \mathcal{M}$ (that is, the set of all points in $\mathrm{S}_{n / 2}^{2} \times \mathrm{S}_{n / 2}^{2}$ where $\mathcal{H}_{1}$ and $\mathcal{H}_{\text {snf }}$ both take the value zero), is a doubly pinched 2-torus in $\mathrm{S}_{n / 2}^{2} \times \mathrm{S}_{n / 2}^{2}$. It follows that the energy momentum map $\mathcal{E} \mathcal{M}$ has monodromy, see [12].

\section{Review of monodromy}

In the past 20 years since the concept of monodromy was introduced into the study of integrable Hamiltonian systems, it has not joined the arsenal of fundamental qualitative ideas used by the physics community. Perhaps the reason for this is that monodromy uses the still insufficiently familiar ideas of global differential geometry. We hope that the following intuitive discussion will explain how one can find and analyze monodromy in an integrable system.

Consider a two degree of freedom Liouville integrable Hamiltonian system. It has two Poisson commuting integrals: the Hamiltonian $H$ and a momentum $J$. The phase curves of this system lie on a subset of a 4-dimensional phase space $P$. Generically, this subset is a 2-dimensional torus $\mathbf{T}^{2}$, but it can also be a point (which is an equilibrium) or a circle $\mathbf{S}^{1}$ (which is a periodic orbit). 
To understand the dynamics of our system, we begin by looking at the energy momentum map

$$
\mathcal{E M}: P \rightarrow \mathbf{R}^{2}: p \rightarrow(H(p), J(p))=(h, j)
$$

Corresponding to each value $(h, j)$ of the map $\mathcal{E M}$ is a fiber $\mathcal{E} \mathcal{M}^{-1}(h, j)$, which is the set of all points in phase space for which the value of $\mathcal{E M}$ is the given value $(h, j)$. We will assume that $(h, j)$ is a regular value of the energy momentum map and that the $(h, j)$-level set of $\mathcal{E M}$ is compact and connected. Then the fiber $\mathcal{E} \mathcal{M}^{-1}(h, j)$ is a smooth 2-dimensional torus $\mathrm{T}_{(h, j)}^{2}$. (This fiber is always a smooth 2-dimensional manifold with no boundary. Since it is compact, by the Arnol'd-Liouville theorem [31] its connected components are 2-dimensional tori.)

What we want to do is to describe how these fibers fit together as $(h, j)$ runs over a parameterized subset of the set of regular values. Suppose that this set of regular values is a small open 2-disc $D$ in the range of the energy momentum map. The action-angle coordinate theorem states that $\mathcal{E M}^{-1}(D)$ (which is the union of 2-tori $\mathrm{T}_{(h, j)}^{2}$ where $(h, j)$ runs over $\left.D\right)$ has the topology of $D \times \mathbf{T}^{2}$. In other words

$$
\mathcal{E} \mathcal{M}^{-1}(D) \rightarrow D: \mathrm{T}_{(h, j)}^{2} \rightarrow(h, j)
$$

is a trivial bundle over $D$ with total space $\mathcal{E} \mathcal{M}^{-1}(D)$, fiber $\mathbf{T}^{2}$, and base space $D$.

This simple geometric situation is greatly complicated if the 2-disc $D$ contains a critical value $(h, j)_{\text {crit }}$ and the punctured disc $D^{*}=D-\left\{(h, j)_{\text {crit }}\right\}$ lies in the set of regular values in the image of $\mathcal{E} \mathcal{M}$. When we are in this situation we say that the critical value $(h, j)_{\text {crit }}$ is isolated. Under quite general conditions the singular fiber $F=\mathcal{E M}^{-1}\left((h, j)_{\text {crit }}\right)$ is a "pinched" 2-torus shown in fig. 1. Dynamically, a singly pinched 2-torus is a homoclinic connection of stable and unstable manifolds of the pinch point, whereas a doubly pinched one is a heteroclinic connection of the stable and unstable manifolds of the two pinch points.

When the singular fiber $F$ is a pinched 2-torus, the foliation of $\mathcal{E} M^{-1}\left(D^{*}\right)$ by the 2-tori $\mathcal{E M}^{-1}(h, j)$ with $(h, j) \in D^{*}$ is nontrivial [12]. This can be understood by taking a circle $\Gamma$ in $D^{*}$ and looking at the bundle $\Pi: \mathcal{E M}^{-1}(\Gamma) \rightarrow \Gamma$ over $\Gamma$. Geometrically every 2 -torus bundle over a circle can be obtained by the following construction. Consider the trivial 2-torus bundle $[0,1] \times \mathbf{T}^{2}$ over the closed interval $[0,1]$. Form a circle in the base of the bundle by identifying the end points of $[0,1]$ to a single point. To obtain a 2-torus bundle over this circle identify the end 2 -tori $\{0\} \times \mathbf{T}^{2}$ and $\{1\} \times \mathbf{T}^{2}$ by an invertible 

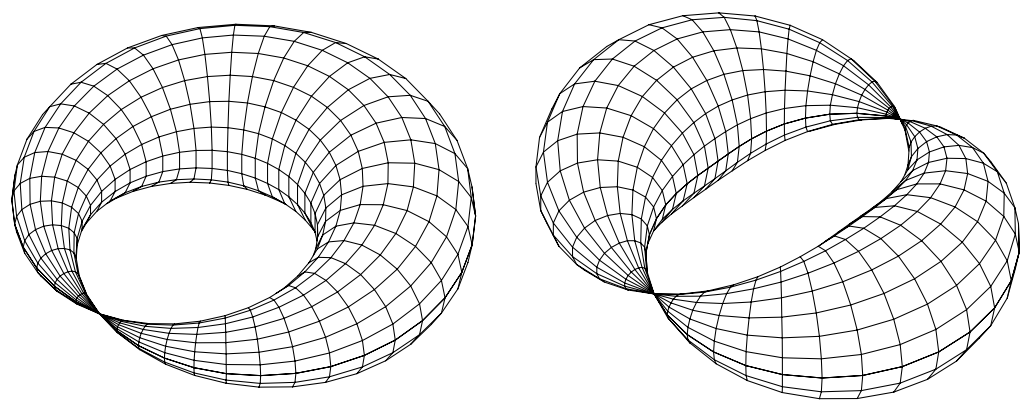

Fig. 1. Singly and doubly pinched torus: a homoclinic and heteroclinic connection of stable and unstable manifolds.

map $M: \mathbf{T}^{2} \rightarrow \mathbf{T}^{2}$, called the monodromy map. This map glues the end tori together after giving them a twist. For a lower dimensional example of this twisting construction, think of a cylinder and a Möbius band. Start with a product of $[0,1]$ and an open interval (which is a trivial bundle over $[0,1]$ ). A cylinder is formed by gluing the open intervals over the end points by the identity map, while the Möbius band is formed by using minus the identity as the gluing map.

We return to considering our 2-torus bundle $\Pi$ over the circle $\Gamma$. Here is a theoretical method for computing its monodromy map. Cut the circle $\Gamma$ at a point $p$ and think of it as an interval whose closure $\mathcal{I}$ has end points $p_{0}$ and $p_{1}$. Cover the interval $\mathcal{I}$ by a finite set of pairwise overlapping intervals $\mathcal{I}_{i}$ on which the local actions given by the action-angle coordinate theorem are defined. On the overlap $\mathcal{I}_{i} \cap \mathcal{I}_{i+1}$ adjust the actions so that they agree. As a result of this construction we have found the values $\left(j_{1}^{p_{1}}, j_{2}^{p_{1}}\right)$ of the actions at $p_{1}$ starting with their values at $\left(j_{1}^{p_{0}}, j_{2}^{p_{0}}\right)$ at $p_{0}$ by following the curve $\Gamma-\{p\}$. Care is needed because, as functions on the set of regular values of the energy momentum map, the actions $\left(j_{1}, j_{2}\right)$ may be multi-valued. The actions $\left(j_{1}^{p_{i}}, j_{2}^{p_{i}}\right)$ label the 2-torus $\mathcal{E} \mathcal{M}^{-1}\left(p_{i}\right)$ for $i=1,2$. We think of the 2-torus $\mathcal{E} \mathcal{M}^{-1}\left(p_{0}\right)$ as the space $\mathbf{R}^{2} / L_{p_{0}}$, where $L_{p_{0}}$ is the lattice generated by evaluating the Hamiltonian vector fields $X_{j_{i}^{p_{0}}}$ corresponding to the action $j_{i}^{p_{0}}$ at the point $p_{0}$. In other words, two vectors in $\mathbf{R}^{2}$ represent the same point on the 2-torus $\mathcal{E} \mathcal{M}^{-1}\left(p_{0}\right)$ if their coordinates differ by some integer linear combination of vectors in $L_{p_{0}}$. Similarly, the 2-torus $\mathcal{E} \mathcal{M}^{-1}\left(p_{1}\right)$ is $\mathbf{R}^{2} / L_{p_{1}}$. Consider the invertible linear map $M$ which assigns to the generators of $L_{p_{0}}$ the generators of $L_{p_{1}}$. The map $M$ is given by an integer $2 \times 2$ matrix with determinant 1 , which maps the 2-torus $\mathcal{E} \mathcal{M}^{-1}\left(p_{0}\right)$ onto the 2-torus $\mathcal{E} \mathcal{M}^{-1}\left(p_{1}\right)$. Of course the torus $\mathcal{E} \mathcal{M}^{-1}\left(p_{1}\right)$ is the same as the torus $\mathcal{E} \mathcal{M}^{-1}\left(p_{0}\right)$. Thus $M$ is the monodromy map of the bundle $\Pi$. It is a theorem [32] that $M$ has the form $\left(\begin{array}{ll}1 & k \\ 0 & 1\end{array}\right)$, where $k$ is the number of pinch points of the singular fiber $F$. From the above discussion it is clear that the monodromy matrix determines the global geometry of the 2-torus bundle around a pinched 2-torus singular fiber $F$ of the energy momentum mapping. 


\section{Review of first normal form}

This section reviews the familiar grounds for obtaining the first normal form. Our treatment follows [20] and explains the details of the field strength scaling. More explanation of the computation of the first normal form, its finite symmetries and its expression in terms of the $\mathrm{SO}(4)$ symmetry generators can be found in $[20,23]$.

\subsection{Regularization and rescaling}

In order to perform normalization one needs to regularize the Hamiltonian (1) so that its bounded orbits are defined for all time. Intuitively speaking, regularization removes the $1 / r$ singularity from the Kepler Hamiltonian.

First we fix a value $E<0$ of the energy. Rescale length and momentum by $(Q, P) \rightarrow\left(C^{-1} Q, C P\right)$ so that the effective charge $C$ in (1) becomes 1 . Then rescaling time by $t \rightarrow C^{2} t$, the Hamiltonian (1) becomes

$$
0=\frac{1}{2} \boldsymbol{P}^{2}-\frac{1}{|Q|}-\frac{E}{C^{2}}+\frac{F}{C^{3}} Q_{2}+\frac{G}{2 C^{2}}\left(Q_{2} P_{3}-Q_{3} P_{2}\right)+\frac{G^{2}}{8 C^{4}}\left(Q_{2}^{2}+Q_{3}^{2}\right) .
$$

Next define a new time scale by $d t \rightarrow \frac{1}{|Q|} d t$. The above expression becomes

$$
\begin{aligned}
0= & \frac{1}{2}|Q|\left(P^{2}+\frac{-2 E}{C^{2}}\right)-1+\frac{F}{C^{3}} Q_{2}|Q|+\frac{G}{2 C^{2}}\left(Q_{2} P_{3}-Q_{3} P_{2}\right)|Q| \\
& +\frac{G^{2}}{8 C^{4}}\left(Q_{2}^{2}+Q_{3}^{2}\right)|Q| .
\end{aligned}
$$

We now regularize (2b) using the method of Kustaanheimo and Stiefel (KS). The KS method lifts the phase space $T_{0} \mathbf{R}^{3}=\left(\mathbf{R}^{3}-\{0\}\right) \times \mathbf{R}^{3}$ [with canonical coordinates $(Q, P)]$ to the larger phase space $T_{0} \mathbf{R}^{4}=\left(\mathbf{R}^{4}-\{0\}\right) \times \mathbf{R}^{4}$ [with canonical coordinates $(q, p)]$ using the mapping

$$
\begin{aligned}
& \mathrm{KS}: T_{0} \mathbf{R}^{4} \rightarrow T_{0} \mathbf{R}^{3}: \\
&(q, p) \rightarrow\left(M_{\mathrm{KS}}(q), \frac{1}{r} M_{\mathrm{KS}}(p)\right)=(Q, 0, P, 0) . \\
& \text { Here } M_{\mathrm{KS}}=\left(\begin{array}{rrrr}
q_{1} & -q_{2} & -q_{3} & q_{4} \\
q_{2} & q_{1} & -q_{4} & -q_{3} \\
q_{3} & q_{4} & q_{1} & q_{2} \\
q_{4} & -q_{3} & q_{2} & -q_{1}
\end{array}\right) \text { and } r=|Q|=q^{2} \text {. In defining the KS map }
\end{aligned}
$$


we have required that

$$
\zeta=q_{1} p_{4}-q_{2} p_{3}+q_{3} p_{2}-q_{4} p_{1}=0
$$

Using the KS map, equation (2b) becomes

$$
\begin{aligned}
1=\frac{1}{2}\left(\frac{1}{4} p^{2}+\frac{-2 E}{C^{2}} q^{2}\right) & +2 \frac{F}{C^{3}}\left(q_{1} q_{2}-q_{3} q_{4}\right) q^{2}+\frac{1}{2} \frac{G}{C^{2}}\left(q_{2} p_{3}-q_{3} p_{2}\right) q^{2} \\
& +\frac{1}{8} \frac{G^{2}}{C^{4}}\left(q_{1}^{2}+q_{4}^{2}\right)\left(q_{2}^{2}+q_{3}^{2}\right) q^{2} .
\end{aligned}
$$

After rescaling the variables $q$ and $p$ by $(q, p) \rightarrow\left(\frac{q}{\sqrt{\omega}}, p \sqrt{\omega}\right)$, where $\sqrt{\omega}=$ $\frac{2 \sqrt{-2 E}}{C}$, and rescaling time by $t \rightarrow \frac{\omega}{4} t$, equation $(5)$ becomes

$$
\begin{aligned}
\frac{4}{\omega}=H=\frac{1}{2}\left(p^{2}+q^{2}\right) & +\frac{1}{3} f\left(q_{1} q_{2}-q_{3} q_{4}\right)^{2} q^{2}+\frac{1}{2} g\left(q_{2} p_{3}-q_{3} p_{2}\right) q^{2} \\
& +\frac{1}{8} g^{2}\left(q_{1}^{2}+q_{4}^{2}\right)\left(q_{2}^{2}+q_{3}^{2}\right) q^{2} .
\end{aligned}
$$

Writing the scaled field parameters as

$$
f=3 F\left(\frac{2}{C \omega}\right)^{3}=\varepsilon \beta \quad \text { and } \quad g=G\left(\frac{2}{C \omega}\right)^{2}=\varepsilon \alpha
$$

where $\alpha$ and $\beta$ are two dimensionless parameters satisfying

$$
\alpha \geq 0, \quad \beta \geq 0, \quad \alpha^{2}+\beta^{2}=1
$$

and $\varepsilon$ is a smallness parameter [33], the Hamiltonian $H(6)$ can be written as

$$
\begin{aligned}
H(q, p)=\frac{1}{2}\left(p^{2}+q^{2}\right)+ & \varepsilon\left(\beta\left(q_{1} q_{2}-q_{3} q_{4}\right)^{2}+\alpha\left(q_{2} p_{3}-q_{3} p_{2}\right)\right) q^{2} \\
& +\frac{1}{2} \varepsilon^{2}\left(\frac{1}{4} \alpha^{2}\left(q_{1}^{2}+q_{4}^{2}\right)\left(q_{2}^{2}+q_{3}^{2}\right) q^{2}\right) \\
= & H_{0}+\varepsilon H_{1}+\frac{1}{2} \varepsilon^{2} H_{2},
\end{aligned}
$$

which is a perturbation of the $1: 1: 1: 1$ harmonic oscillator. 


\subsection{Normalization and reduction}

Having written the Hamiltonian (8) as a perturbation of the harmonic oscillator $H_{0}$, we can carry out its normalization using standard Lie series methods. This normalization procedure gives a canonical coordinate change on $T \mathbf{R}^{4}$ for which the transformed Hamiltonian Poisson commutes with $H_{0}$ up through second order terms in $\varepsilon$. The truncated normalized Hamiltonian

$$
\mathcal{H}_{\text {fnf }}=H_{0}+\varepsilon \mathcal{H}_{1}+\frac{1}{2} \varepsilon^{2} \mathcal{H}_{2}
$$

also Poisson commutes with the KS integral $\zeta(4)$ because the normalizing coordinate change commutes with the $\mathbf{S}^{1}$ symmetry of $H(8)$ generated by the flow of $X_{\zeta}$. Thus $\mathcal{H}_{\mathrm{fnf}}$ is invariant under the $\mathbf{T}^{2}$ symmetry generated by $H_{0}$ and $\zeta$.

The algebra of polynomials on $T \mathbf{R}^{4}$ which are invariant under this $\mathbf{T}^{2}$ action is generated by

$$
\begin{aligned}
K_{1} & =\frac{1}{4}\left[p_{2}^{2}+q_{2}^{2}+p_{3}^{2}+q_{2}^{2}-\left(p_{1}^{2}+q_{1}^{2}\right)-\left(p_{4}^{2}+q_{4}^{2}\right)\right], \\
K_{2} & =\frac{1}{4}\left(p_{3} p_{4}-q_{1} q_{2}-p_{1} p_{2}+q_{3} q_{4}\right), \\
K_{3} & =-\frac{1}{2}\left(q_{1} q_{3}+q_{2} q_{4}+p_{1} p_{3}+p_{2} p_{4}\right), \\
L_{1} & =\frac{1}{2}\left(q_{2} p_{3}-q_{3} p_{2}+q_{1} p_{4}-q_{4} p_{1}\right), \\
L_{2} & =\frac{1}{2}\left(q_{2} p_{4}+q_{3} p_{1}-q_{1} p_{3}-q_{4} p_{2}\right), \\
L_{3} & =\frac{1}{2}\left(q_{1} p_{2}+q_{3} p_{4}-q_{2} p_{1}-q_{4} p_{3}\right),
\end{aligned}
$$

together with $H_{0}$ and $\zeta$. The vectors $K=\left(K_{1}, K_{2}, K_{3}\right)$ and $L=\left(L_{1}, L_{2}, L_{3}\right)$ are nothing but the modified eccentricity [34] and angular momentum vectors for the Kepler Hamiltonian written in terms of the KS variables $(q, p)$. The above $\mathbf{T}^{2}$-invariants satisfy the relations

$$
K \cdot K+L \cdot L=\frac{1}{4} H_{0}^{2} \quad \text { and } \quad K \cdot L=0 .
$$

Thus the space of $\mathbf{T}^{2}$ orbits on $H_{0}^{-1}(2 n) \cap \zeta^{-1}(0)$ is defined by

$$
K^{2}+L^{2}=n^{2} \quad \text { and } \quad K \cdot L=0 .
$$

Since (11a) is equivalent to

$$
(K+L)^{2}=n^{2} \quad \text { and } \quad(K-L)^{2}=n^{2},
$$


the $\mathbf{T}^{2}$-orbit space is the product of two 2 -spheres $S_{n / 2}^{2} \times S_{n / 2}^{2}$. The Poisson structure on $S_{n / 2}^{2} \times S_{n / 2}^{2}$ is determined by the so(4) relations

$$
\left\{L_{i}, L_{j}\right\}=\varepsilon_{i j k} L_{k}, \quad\left\{K_{i}, K_{j}\right\}=\varepsilon_{i j k} L_{k}, \quad \text { and } \quad\left\{L_{i}, K_{j}\right\}=\varepsilon_{i j k} K_{k} .
$$

In [20] the first normalized Hamiltonian $\mathcal{H}_{\text {fnf }}$ in $(9)$ is expressed in terms of $\mathbf{T}^{2}$-invariant polynomials restricted to $\mathrm{S}_{n / 2}^{2} \times \mathrm{S}_{n / 2}^{2}$. Rescaling time by $t \rightarrow-t n$ and dropping the additive constant $\left(3 \alpha^{2}-17 \beta^{2} / 9\right) \varepsilon / 4$, we can write $\mathcal{H}_{\text {fnf }}$ as

$$
\begin{aligned}
H_{0}= & 2 \\
\mathcal{H}_{1}= & \alpha L_{1}+\beta K_{2} \\
\mathcal{H}_{2}= & \frac{\alpha^{2}}{4}\left[3 L_{1}^{2}+2 L_{2}^{2}+3 K_{1}^{2}-2 K_{2}^{2}+2\left(L_{3}^{2}-K_{3}^{2}\right)\right] \\
& +\frac{\alpha \beta}{3}\left(7 K_{2} L_{1}-L_{2} K_{1}\right)+\frac{\beta^{2}}{12}\left(17 K_{2}^{2}-3 L_{2}^{2}\right) .
\end{aligned}
$$

\section{Second normal form}

In this section we show how to normalize the Hamiltonian $\mathcal{H}_{\text {fnf }}$ of the first normal form once again using the $\mathbf{S}^{1}$ symmetry generated by $\mathcal{H}_{1}$. We then reduce this $\mathbf{S}^{1}$ symmetry to obtain a one degree of freedom Hamiltonian $\mathcal{H}_{n, c}$ on a possibly singular second reduced phase space $P_{n, c}$. When $c=0$ we analyze the geometry of this one degree of freedom system $\mathcal{H}_{n, 0}$ on the singular space $P_{n, 0}$. We find an open interval of values of the parameter $\alpha$ such that the energy momentum map $\left(\mathcal{H}_{\mathrm{snf}}, \mathcal{H}_{1}\right)$ has monodromy.

\subsection{Calculation of the second normal form}

In order to calculate the second normal form for $\mathcal{H}_{\text {fnf }}$ in (13), we make its first order term $\mathcal{H}_{1}=\alpha L_{1}+\beta K_{2}$ the first basis element of the so(4) Poisson algebra (12). To do this we use the fact that $\alpha^{2}+\beta^{2}=1$ and define a Poisson automorphism $(L, K) \rightarrow(T, V)$ with

$$
(T, V)=\left(\left(\begin{array}{c}
\alpha L_{1}+\beta K_{2} \\
\alpha L_{2}-\beta K_{1} \\
L_{3}
\end{array}\right),\left(\begin{array}{c}
\beta L_{2}+\alpha K_{1} \\
\alpha K_{2}-\beta L_{1} \\
K_{3}
\end{array}\right)\right)
$$


Of course, the Poisson brackets for the components of $T$ and $V$ are the same as in (12) with $L$ and $K$ replaced by $T$ and $V$ respectively. We can also work directly with the Poisson algebra generated by the components of $x=\frac{1}{2}(T+V)$ and $y=\frac{1}{2}(T-V)$, namely

$$
\begin{array}{rlr}
x_{1}=\frac{T_{1}+V_{1}}{2}, & x_{2}=\frac{T_{2}+V_{2}}{2}, & x_{3}=\frac{T_{3}+V_{3}}{2}, \\
y_{1}=\frac{T_{1}-V_{1}}{2}, & y_{2}=\frac{T_{2}-V_{2}}{2}, & y_{3}=\frac{T_{3}-V_{3}}{2} .
\end{array}
$$

In terms of these variables, the first reduced phase space $\mathrm{S}_{n / 2}^{2} \times \mathrm{S}_{n / 2}^{2}$ is defined by the Casimirs

$$
x_{1}^{2}+x_{2}^{2}+x_{3}^{2}=\frac{n^{2}}{4} \quad \text { and } \quad y_{1}^{2}+y_{2}^{2}+y_{3}^{2}=\frac{n^{2}}{4},
$$

and the Poisson bracket satisfies the $s o(3) \times \operatorname{so}(3)$ relations

$$
\left\{x_{i}, x_{j}\right\}=\varepsilon_{i j k} x_{k}, \quad\left\{y_{i}, y_{j}\right\}=\varepsilon_{i j k} y_{k}, \quad \text { and } \quad\left\{x_{i}, y_{j}\right\}=0
$$

After dropping the constant $H_{0}=2$, rescaling the time by $t \rightarrow \varepsilon t$, and then changing to variables $(x, y)$, the first normalized Hamiltonian (9) up to first order becomes

$$
\mathcal{H}_{\text {fnf }}=\mathcal{H}_{1}+\frac{\varepsilon}{2} \mathcal{H}_{2}=T_{1}+\frac{\varepsilon}{2} \mathcal{H}_{2}=\left(x_{1}+y_{1}\right)+\frac{\varepsilon}{2} \mathcal{H}_{2}
$$

where

$$
\begin{aligned}
\mathcal{H}_{2}= & \frac{1}{3}\left(2 \alpha^{4}-\alpha^{2}+7 / 2\right)\left(x_{1}^{2}+y_{1}^{2}\right)+\frac{2}{3} \alpha^{2}\left(1-\alpha^{2}\right)\left(x_{2}^{2}+y_{2}^{2}\right) \\
& +\frac{1}{3} \alpha \beta\left(1-4 \alpha^{2}\right)\left(x_{1} x_{2}-y_{1} y_{2}\right)+\frac{2}{3} \alpha \beta\left(x_{2} y_{1}-x_{1} y_{2}\right) \\
& +2 \alpha^{2}\left(x_{2} y_{2}+x_{3} y_{3}\right)+\frac{10}{3}\left(1-\alpha^{2}\right) x_{1} y_{1} .
\end{aligned}
$$

The vector field

$$
\begin{aligned}
X_{\mathcal{H}_{1}} & =-T_{3} \frac{\partial}{\partial T_{2}}+T_{2} \frac{\partial}{\partial T_{3}}-V_{3} \frac{\partial}{\partial V_{2}}+V_{2} \frac{\partial}{\partial V_{3}} \\
& =-x_{3} \frac{\partial}{\partial x_{2}}+x_{2} \frac{\partial}{\partial x_{3}}-y_{3} \frac{\partial}{\partial y_{2}}+y_{2} \frac{\partial}{\partial y_{3}}
\end{aligned}
$$


has flow given by

$$
\varphi_{t}(x, y)=\left(R_{t} x, R_{t} y\right), \quad R_{t}=\left(\begin{array}{ccc}
1 & 0 & 0 \\
0 & \cos t & -\sin t \\
0 & \sin t & \cos t
\end{array}\right) .
$$

In other words, $\varphi_{t}$ defines an $\mathbf{S}^{1}$ action on $\mathbf{R}^{3} \times \mathbf{R}^{3}$ which satisfies $\varphi_{2 \pi}=\mathrm{id}$ and leaves the first reduced phase space $\mathrm{S}_{n / 2}^{2} \times \mathrm{S}_{n / 2}^{2}$ invariant. Thus we may normalize $\mathcal{H}_{\mathrm{fnf}}$ a second time. This can be done by simple averaging of $\mathcal{H}_{2}$ along the integral orbits of $X_{\mathcal{H}_{1}}$ given by $\varphi_{t}$, namely

$$
\overline{\mathcal{H}}_{2}(x, y)=\frac{1}{2 \pi} \int_{0}^{2 \pi} \mathcal{H}_{2}\left(\varphi_{t}(x, y)\right) d t
$$

Thus to first order the second normalized Hamiltonian is

$$
\mathcal{H}_{\mathrm{snf}}=\mathcal{H}_{1}+\frac{\varepsilon}{2} \overline{\mathcal{H}}_{2}
$$

with

$$
\begin{aligned}
\overline{\mathcal{H}}_{2}(x, y)= & \frac{1}{3}\left(2 \alpha^{4}-\alpha^{2}+\frac{7}{2}\right)\left(x_{1}^{2}+y_{1}^{2}\right)+\frac{1}{3} \alpha^{2} \beta^{2}\left(x_{2}^{2}+x_{3}^{2}+y_{2}^{2}+y_{3}^{2}\right) \\
& +\frac{10}{3} \beta^{2} x_{1} y_{1}+2 \alpha^{2}\left(x_{2} y_{2}+x_{3} y_{3}\right) .
\end{aligned}
$$

This can be further simplified using (16) and (7) to

$$
\begin{aligned}
\overline{\mathcal{H}}_{2}(x, y)= & \left(\alpha^{4}-\frac{2}{3} \alpha^{2}+\frac{7}{6}\right)\left(x_{1}^{2}+y_{1}^{2}\right)+\frac{n^{2}}{6} \alpha^{2} \beta^{2} \\
& +\frac{10}{3} \beta^{2} x_{1} y_{1}+2 \alpha^{2}\left(x_{2} y_{2}+x_{3} y_{3}\right) .
\end{aligned}
$$

The normal form $\mathcal{H}_{\mathrm{snf}}$ retains only those terms $\pi(x, y)$ of $\mathcal{H}_{2}$ [a homogeneous polynomial in $(x, y)$ of degree 2] which Poisson commute with $\mathcal{H}_{1}$, that is for which $X_{\mathcal{H}_{1}}(\pi(x, y))=0$. Since $\overline{\mathcal{H}}_{2}$ is constant on the integral curves of $X_{\mathcal{H}_{1}}$, it follows that $\mathcal{H}_{1}$ is a second integral of $X_{\mathcal{H}_{\text {snf }}}$. Thus $\left(\mathcal{H}_{\text {snf }}, \mathcal{H}_{1}\right)$ is a Liouville integrable system on the first reduced phase space $S_{n / 2}^{2} \times S_{n / 2}^{2}$ with coordinates $(x, y)$ and Poisson bracket (17). The energy momentum map for this integrable system is

$$
\mathcal{E} \mathcal{M}: \mathrm{S}_{n / 2}^{2} \times \mathrm{S}_{n / 2}^{2} \rightarrow \mathbf{R}^{2}: p \rightarrow\left(\mathcal{H}_{\mathrm{snf}}(p), \mathcal{H}_{1}(p)\right)
$$




\subsection{Reduction to one degree of freedom}

Here we analyze the integrable system $\left(\mathcal{H}_{\text {snf }}, \mathcal{H}_{1}\right)$ by reducing the $\mathbf{S}^{1}$ symmetry generated by $\mathcal{H}_{1}$ using the method of singular reduction $[2,21]$. We thereby obtain a one degree of freedom system.

\subsubsection{Second reduced phase space}

We first use invariant theory to construct the second reduced space. The algebra of polynomials on $\mathbf{R}^{3} \times \mathbf{R}^{3}$ which are invariant under the $\mathbf{S}^{1}$ action defined by $\varphi_{t}(20)$ is generated by

$$
\begin{aligned}
& \pi_{1}=x_{1}-y_{1}=V_{1} \\
& \pi_{2}=4\left(x_{2} y_{2}+x_{3} y_{3}\right)=T_{2}^{2}+T_{3}^{2}-V_{2}^{2}-V_{3}^{2} \\
& \pi_{3}=4\left(x_{3} y_{2}-x_{2} y_{3}\right)=2\left(T_{2} V_{3}-T_{3} V_{2}\right) \\
& \pi_{4}=x_{1}+y_{1}=T_{1} \\
& \pi_{5}=4\left(x_{2}^{2}+x_{3}^{2}\right) \\
& \pi_{6}=4\left(y_{2}^{2}+y_{3}^{2}\right)
\end{aligned}
$$

subject to the relation

$$
\pi_{2}^{2}+\pi_{3}^{2}=\pi_{5} \pi_{6}, \quad \pi_{5} \geq 0, \quad \pi_{6} \geq 0 .
$$

Equation (25) defines the space of $\varphi_{t}$ orbits on $\mathbf{R}^{3} \times \mathbf{R}^{3}$. To find an explicit defining relation for the second reduced phase space we note that the $c$-level set of $\mathcal{H}_{1}$, which is given by

$$
\mathcal{H}_{1}=T_{1}=x_{1}+y_{1}=c, \quad|c| \leq n,
$$

and (16), is a $\varphi_{t}$-invariant submanifold $M_{c}$ of $\mathrm{S}_{n / 2}^{2} \times \mathrm{S}_{n / 2}^{2} \subset \mathbf{R}^{3} \times \mathbf{R}^{3}$. The second reduced phase space $P_{n, c}$ is the space of $\varphi_{t}$ orbits on $M_{c}$ and is defined in terms of invariant polynomials (24) by

$$
\begin{aligned}
\pi_{4} & =c \\
\pi_{5} & =n^{2}-\left(\pi_{1}+\pi_{4}\right)^{2} \\
\pi_{6} & =n^{2}-\left(\pi_{4}-\pi_{1}\right)^{2} \\
\pi_{2}^{2}+\pi_{3}^{2} & =\pi_{5} \pi_{6}, \quad \pi_{5} \geq 0, \pi_{6} \geq 0 .
\end{aligned}
$$

[The first three equations above come from expressing the defining equations (26) and (16) of $M_{c}$ in terms of invariant polynomials (24). These equations 


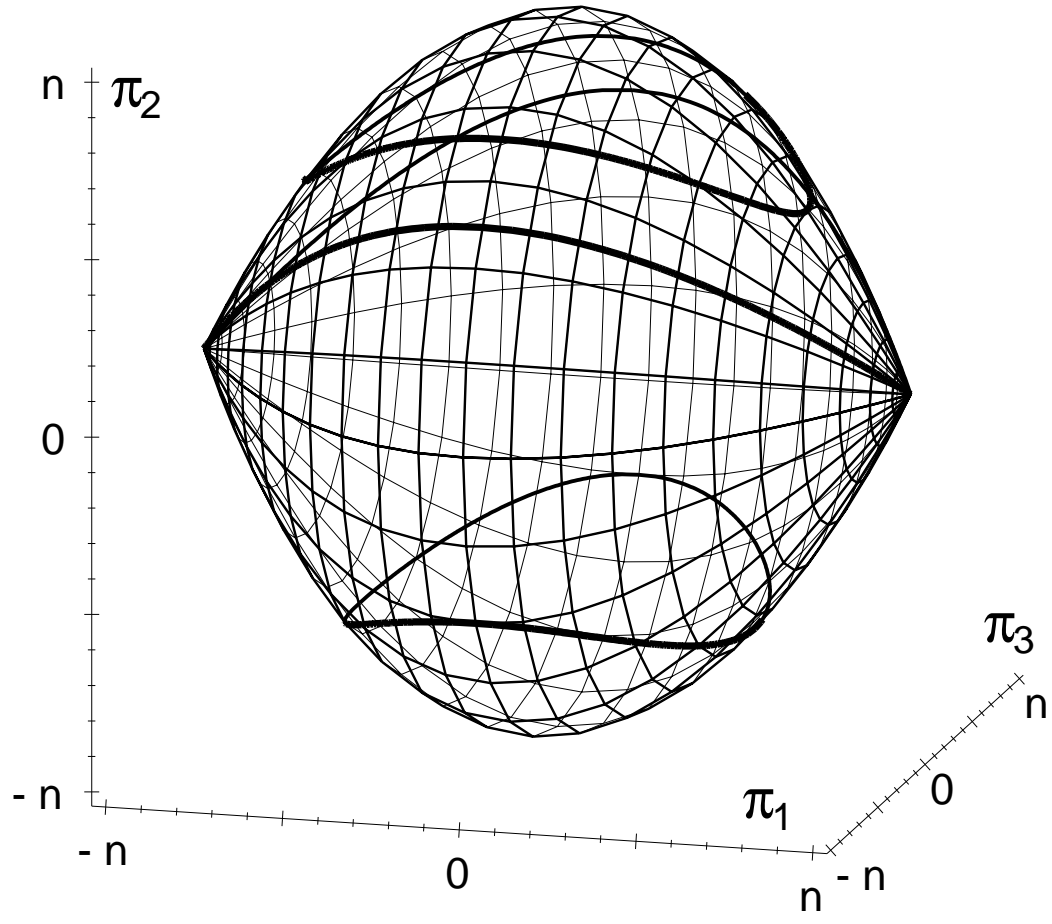

Fig. 2. The second reduced phase space $P_{n, 0}$

are complemented by the relation (25).] Using the relations in (27) to eliminate the variables $\pi_{4}, \pi_{5}$, and $\pi_{6}$, we see that $P_{n, c}$ is the semialgebraic variety defined in $\mathbf{R}^{3}$ with coordinates $\left(\pi_{1}, \pi_{2}, \pi_{3}\right)$ by

$$
\pi_{2}^{2}+\pi_{3}^{2}=\left[(n-c)^{2}-\pi_{1}^{2}\right]\left[(n+c)^{2}-\pi_{1}^{2}\right]
$$

The values of $\pi_{1}, \pi_{2}$, and $\pi_{3}$ in (28a) are subject to the restrictions

$$
\left|\pi_{1}\right| \leq n-|c|, \quad\left|\pi_{2}\right| \leq n^{2}-c^{2}, \quad\left|\pi_{3}\right| \leq n^{2}-c^{2} .
$$

(The first restriction follows from the fact that for any $|c| \leq n$ and $\left|\pi_{1}\right| \leq n$ [use (16)] the two factors on the right hand side of (28a) cannot be both negative and hence they should be both positive.) From (28) we can see that when $0<|c|<n$, the second reduced phase space is a smooth 2-sphere; when $|c|=n$ it is a point; when $c=0$ it is a topological 2-sphere with two conical singular points shown in fig. 2 . The reason why $P_{n, 0}$ has two singular points is that the $\mathbf{S}^{1}$ action $\varphi_{t}$ on $M_{0}$ has two fixed points $(x, y)=\frac{n}{2}( \pm 1,0,0, \mp 1,0,0)$. [The two other fixed points of the $\varphi_{t}$ action on $\mathrm{S}_{n / 2}^{2} \times \mathrm{S}_{n / 2}^{2}$ are $(x, y)= \pm \frac{n}{2}(1,0,0,1,0,0)$ corresponding to $P_{n, \pm n}$. See $[20,23]$ for more details.] 


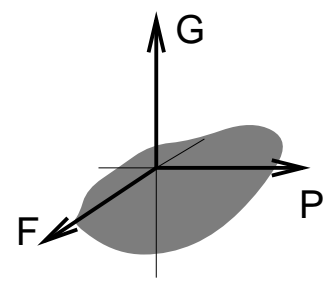

$\sigma_{2}$
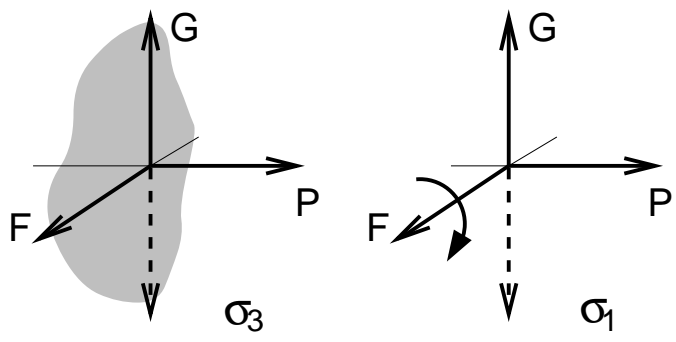

Fig. 3. Action of the symmetry operations of the $\mathbf{Z}_{2} \times \mathbf{Z}_{2}$ finite symmetry group of the hydrogen atom in orthogonal fields on the vectors of electric and magnetic fields $F$ and $G$. (Position of $G$ obtained without momentum reversal which sends $G \rightarrow-G$ is shown by the dashed line.)

\section{D.2 Reduction of finite symmetries}

As discussed in [20], the original Hamiltonian $H$ (1) has two distinct $\mathbf{Z}_{2}$ symmetries: one given by the composition of momentum reversal $(Q, P) \rightarrow$ $(Q,-P)$ and rotation by $\pi$ around axis $Q_{2}$ of the electric field $F$

$$
\sigma_{1}:(Q, P) \rightarrow\left(-Q_{1}, Q_{2},-Q_{3}, P_{1},-P_{2}, P_{3}\right)
$$

and the other given by a reflection in the plane orthogonal to axis $Q_{1}$ of the magnetic field $G$

$$
\sigma_{2}:(Q, P) \rightarrow\left(-Q_{1}, Q_{2}, Q_{3},-P_{1}, P_{2}, P_{3}\right)
$$

The two $\mathbf{Z}_{2}$ actions commute and the total finite symmetry group of (1) is the group $\mathbf{Z}_{2} \times \mathbf{Z}_{2}$ of order four. Its third nontrivial operation is

$$
\sigma_{3}:(Q, P) \rightarrow\left(Q_{1}, Q_{2},-Q_{3},-P_{1},-P_{2}, P_{3}\right)
$$

which is the composition of the momentum reversal and reflection in the plane spanned by the electric and magnetic field vectors. These symmetries are illustrated in fig. 3. Tracing these symmetries through the two reduction steps, we find that their action on the invariants $\pi_{k}$ in (24) (and thus on the second reduced phase space $\left.P_{n, c}\right)$ is given by

$$
\begin{aligned}
& \sigma_{1}:\left(\pi_{1}, \pi_{2}, \pi_{3}\right) \rightarrow\left(-\pi_{1}, \pi_{2}, \pi_{3}\right), \\
& \sigma_{2}:\left(\pi_{1}, \pi_{2}, \pi_{3}\right) \rightarrow\left(-\pi_{1}, \pi_{2},-\pi_{3}\right), \\
& \sigma_{3}:\left(\pi_{1}, \pi_{2}, \pi_{3}\right) \rightarrow\left(\pi_{1}, \pi_{2},-\pi_{3}\right) .
\end{aligned}
$$

The orbit map $\left(\pi_{1}, \pi_{2}, \pi_{3}\right) \rightarrow\left(w, \pi_{2}, \pi_{3}\right)$ of the $\mathbf{Z}_{2}$ subgroup generated by (29a) can be defined as

$$
w=(n-|c|)^{2}-\pi_{1}^{2}
$$



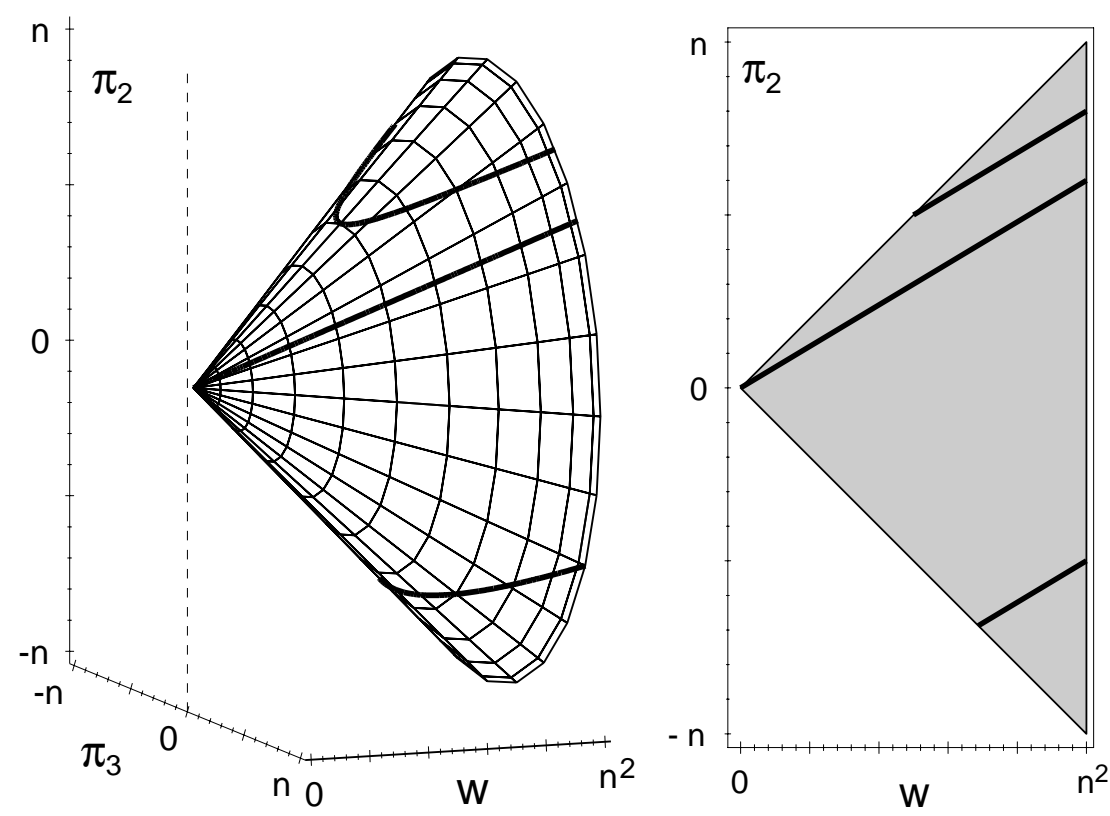

Fig. 4 . The variety $V_{n, 0}$ (left) obtained as the orbit space of the $\mathbf{Z}_{2}$ action (29a) on the second reduced phase space $P_{n, 0}$. Fully symmetry reduced phase space $V_{n, 0}^{0}$ (right) obtained as the orbit space of the $\mathbf{Z}_{2} \times \mathbf{Z}_{2}$ action (29) on the second reduced phase space $P_{n, 0}$ in Fig. 2 . $V_{n, 0}^{0}$ is a projection of the variety $V_{n, 0}$ (left) on the $\pi_{3}=0$ plane.

Thus the image of $P_{n, c}$ under (30) is the semialgebraic variety $V_{n, c}$ defined in $\mathbf{R}^{3}$ with coordinates $\left(w, \pi_{2}, \pi_{3}\right)$ by

$$
\pi_{2}^{2}+\pi_{3}^{2}=w(w+2 n|c|), \quad 0 \leq w \leq(n-|c|)^{2} .
$$

When $0<|c|<n, V_{n, c}$ is a smooth manifold with boundary at $w=(n-|c|)^{2}$ which is diffeomorphic to a closed 2-disc; when $|c|=n$ it is a point; when $c=0$, the variety $V_{n, 0}$ is a topological closed 2-disc with a conical singular point, see fig. 4 . The remaining $\mathbf{Z}_{2}$ symmetries $(29 \mathrm{c})$ and $(29 \mathrm{~b})$ induce a $\mathbf{Z}_{2}$ action on $V_{n, c}$ generated by

$$
\left(w, \pi_{2}, \pi_{3}\right) \rightarrow\left(w, \pi_{2},-\pi_{3}\right)
$$

The orbit space $V_{n, c}^{0}$ of this $\mathbf{Z}_{2}$ action on $V_{n, c}$ is the $\left\{\pi_{3}=0\right\}$-slice of $V_{n, c}$ (see fig. 4, right), that is,

$$
V_{n, c}^{0}=V_{n, c} \cap\left\{\pi_{3}=0\right\}
$$

We call the space $V_{n, c}^{0}$ the full symmetry reduced space of the second normal form. 


\subsubsection{Reduced second normal form}

The second normal form and the manifold $M_{c}$ are invariant under the action $\varphi_{t}(20)$. The restriction of $\mathcal{H}_{\text {snf }}(22)$ descends to a function $\mathcal{H}_{n, c}$ on $P_{n, c}$, called the reduced second normal form. Furthermore, the function $\mathcal{H}_{n, c}$ is invariant with respect to the $\mathbf{Z}_{2} \times \mathbf{Z}_{2}$ symmetry (29) of the problem and can therefore be regarded as a function $\widetilde{\mathcal{H}}_{n, c}$ on the full symmetry reduced phase space $V_{n, c}^{0}$. In other words, $\mathcal{H}_{n, c}$ depends only on $\mathbf{Z}_{2} \times \mathbf{Z}_{2}$ invariant polynomials $\pi_{1}^{2}$ (or $w$ ) and $\pi_{2}$ [35]. It follows that all we have to do in order to define the function $\widetilde{\mathcal{H}}_{n, c}$ on $V_{n, c}^{0}$ is to express $\mathcal{H}_{\text {snf }}(22)$ in terms of the invariants $\pi_{2}, \pi_{1}$, and $\pi_{4}$, then fix the value of $\pi_{4}=c$, and change to the symmetry coordinate $w$ in $(30)$. In this way we find

$$
\widetilde{\mathcal{H}}_{n, c}=a \pi_{2}+b w, \quad \text { where } \quad a=\alpha^{2}, \quad b=\frac{1}{2}-\alpha^{2}-\alpha^{4} .
$$

Here we have used the relations

$$
x_{1}^{2}+y_{1}^{2}=\frac{1}{2}\left(\pi_{1}^{2}+\pi_{4}^{2}\right), \quad x_{1} y_{1}=\frac{1}{4}\left(\pi_{4}^{2}-\pi_{1}^{2}\right),
$$

have rescaled time $t \rightarrow t / 2$, and have dropped the additive constant

$$
\frac{c^{2}}{6}\left(6 \alpha^{4}-4 \alpha^{2}+7\right)+\frac{n^{2}}{12}\left(4 \alpha^{4}+8 \alpha^{2}-3\right)+\frac{c n}{4} b .
$$

We note that (to our order of the second normal form) this constant term contains all the dependence on the values of integrals $n$ and $c$ of the first and second normal forms. We also note that even at the third order, the second normal form $\widetilde{\mathcal{H}}_{n, c}$ remains linear in $\pi^{2}$ and $w$ [35].

\subsection{Geometric analysis}

We now analyze the geometry of the level sets of the second reduced normal form $\mathcal{H}_{n, c}$ on the second reduced phase space $P_{n, c}$ when $c=0$, that is when $P_{n, c}$ is singular. It suffices to understand the $h$-level sets of $\widetilde{\mathcal{H}}_{n, 0}$ on the full symmetry reduced space $V_{n, 0}^{0}$. There are two qualitatively different possibilities which are given in figs. 5 and 6 . In these figures we also show the corresponding sets on the $\left\{\pi_{3}=0\right\}$-slice of $P_{n, 0}$. This latter representation was used in [21,22]. Furthermore, for the case $|b / a|<1$ the same levels can be seen in figs. 4 and 2 on $V_{n, 0}$ and $P_{n, 0}$ respectively.

We now determine at what values of the parameter $\alpha \in[0,1]$ the slope $|b / a|$ is less than 1 . In other words we want to have the level set system of the kind 

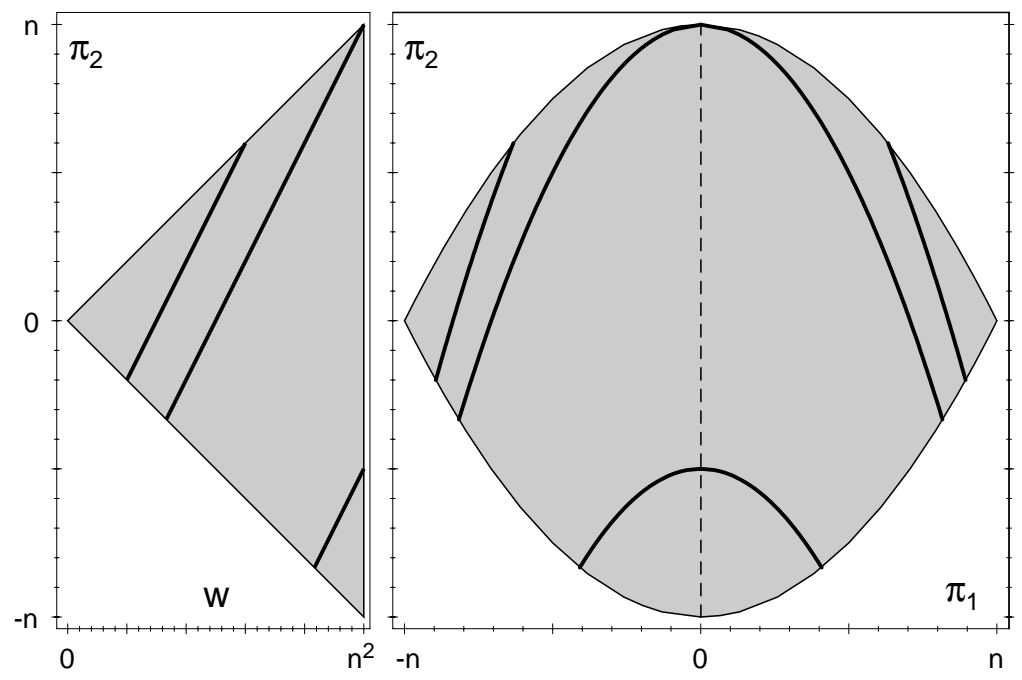

Fig. 5. Constant level sets of $\tilde{\mathcal{H}}_{n, 0}$ on $V_{n, 0}^{0}$ (left) and on $P_{n, 0} \cap\left\{\pi_{3}=0\right\}$ (right) in the case when $|b / a|>1$ (without monodromy).
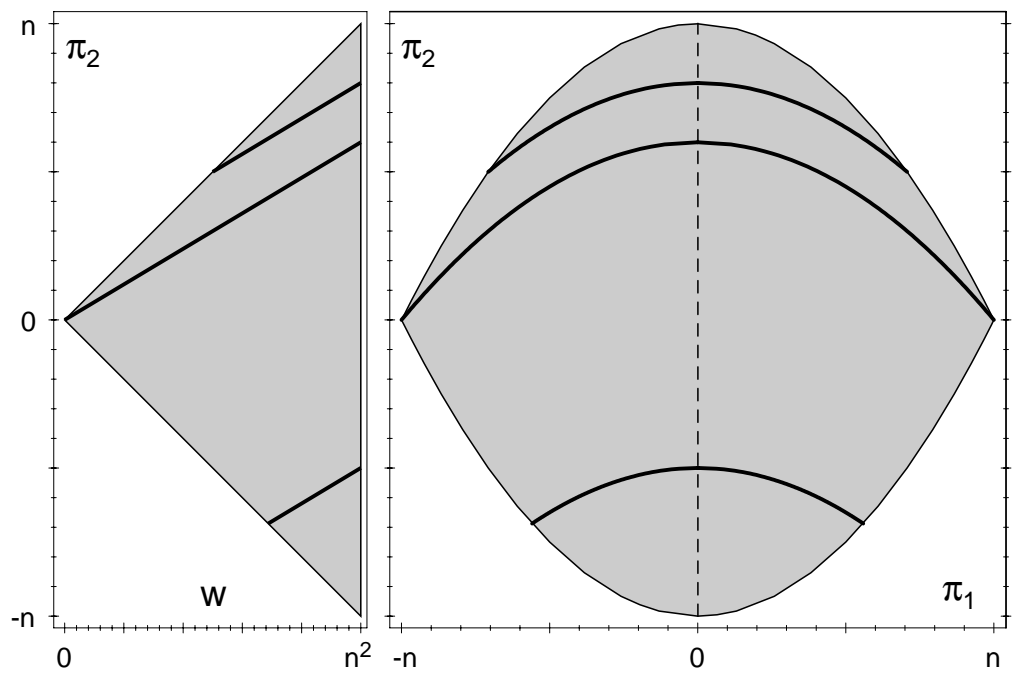

Fig. 6. Constant level sets of $\tilde{\mathcal{H}}_{n, 0}$ on $V_{n, 0}^{0}$ (left) and on $P_{n, 0} \cap\left\{\pi_{3}=0\right\}$ (right) in the case when $|b / a|<1$ (with monodromy). Corresponding levels on $V_{n, 0}$ and $P_{n, 0}$ are shown in Figs. 4 and 2.

shown in fig. 6 where the 0 -level set of $\widetilde{\mathcal{H}}_{n, 0}$ is a closed interval one of whose end points is the singular point $\left(w, \pi_{2}\right)=(0,0)$. Since $a=\alpha^{2}>0$, the condition to be satisfied is $-a<b<a$. We can now see from (32) that the parameter $\alpha>0$ must satisfy

$$
-\alpha^{2}<-\alpha^{4}-\alpha^{2}+1 / 2<\alpha^{2}
$$

The above inequalities become equalities when $\alpha^{2}=\frac{\sqrt{2}}{2}$ and $\alpha^{2}=\frac{\sqrt{6}}{2}-1$. 
Hence $|b / a|<1$ if and only if

$$
\alpha^{2} \in \mathcal{I}=\left(\frac{\sqrt{6}}{2}-1, \frac{\sqrt{2}}{2}\right)
$$

\subsection{Reconstruction and monodromy}

We now show how to reconstruct the geometry of the level sets of the second normal form on $M_{0}$ from the geometry of the level sets of the second reduced Hamiltonian on the second reduced phase space $P_{n, 0}$. We will use the reduction map

$$
\begin{aligned}
\Pi: & M_{0} \subseteq S_{n / 2}^{2} \times S_{n / 2}^{2} \rightarrow P_{n, 0} \subseteq \mathbf{R}^{3}: \\
& (x, y) \rightarrow\left(\pi_{1}(x, y), \pi_{2}(x, y), \pi_{3}(x, y)\right),
\end{aligned}
$$

whose fiber $\Pi^{-1}(p)$ over a point $p$ in $P_{n, 0}$ is a unique $\varphi_{t}$ orbit on $M_{0}$. If $p$ is a nonsingular point of $P_{n, 0}$, then $\Pi^{-1}(p)$ is a circle (that is, a generic $\varphi_{t}$ orbit); whereas if $p$ is a singular point of $P_{n, 0}$ then $\Pi^{-1}(p)$ is a point, which is fixed by the action $\varphi_{t}$.

We carry out our reconstruction only when $|b / a|<1$. The treatment of the other case when $|b / a|>1$ is analogous and is omitted. To follow the discussion please refer to fig. 2 as well as figs. 4 and 6 which illustrate the lift from $V_{n, 0}^{0}$ to $P_{n, 0}$. We begin by considering the case when the level set of $\mathcal{H}_{n, 0}$ is a point $p$. If $p$ is a nonsingular point of $P_{n, 0}$ (a point with $\pi_{1}=0$ and $\left|\pi_{2}\right|=n$ ), then after reconstruction we obtain a periodic orbit $S^{1}=\Pi^{-1}(p)$ of $X_{\mathcal{H}_{1}}$ on $M_{0}$ which is also a periodic orbit of $X_{\mathcal{H}_{\text {snf }}}$ since $\mathcal{H}_{\text {snf }}$ and $\mathcal{H}_{1}$ Poisson commute. These periodic orbits are called relative equilibria of $X_{\mathcal{H}_{\text {snf }}}$. If $p$ is a singular point of $P_{n, 0}$, then after reconstruction we obtain an equilibrium point of $X_{\mathcal{H}_{1}}$ on $M_{0}$ which is also an equilibrium point of $X_{\mathcal{H}_{\text {anf }}}$.

We now look at the 0 -level set of $\mathcal{H}_{n, 0}$. This level set contains the two singular points $p_{0}$ and $p_{1}$ of $P_{n, 0}$. If we remove these points, we obtain two curves $\mathcal{C}_{0}$ and $\mathcal{C}_{1}$ which consist of nonsingular points of $P_{n, 0}$ and which are each topologically an open interval. Over each point on $\mathcal{C}_{i}$ the fiber of the reduction map $\Pi(34)$ is a circle. Since each $\mathcal{C}_{i}$ is contractible in $P_{n, 0}-\left\{p_{0}, p_{1}\right\}$ to a point, it follows that $\Pi^{-1}\left(\mathcal{C}_{i}\right)$ (the set of all points in $M_{0}$ which map by $\Pi$ to points of $\mathcal{C}_{i}$ ) is diffeomorphic to a cylinder $\mathcal{C}_{i} \times S^{1}$. Thus $\Pi^{-1}\left(\mathcal{C}_{i} \cup\left\{p_{0}, p_{1}\right\}\right)$ is a cylinder with each of its ends pinched to a point. The reconstruction $\Pi^{-1}\left(\mathcal{H}_{n, 0}^{-1}(0)\right)$ in $M_{0}$ of the 0 -level of $\mathcal{H}_{n, 0}$ on $P_{n, 0}$ is the union of two pinched cylinders with their end points identified two at a time to two distinct points. In other words, after reconstruction, the 0 -level set of the second reduced Hamiltonian on the second reduced space is a doubly pinched 2-torus in $M_{0}$ (see fig. 1 , right). 


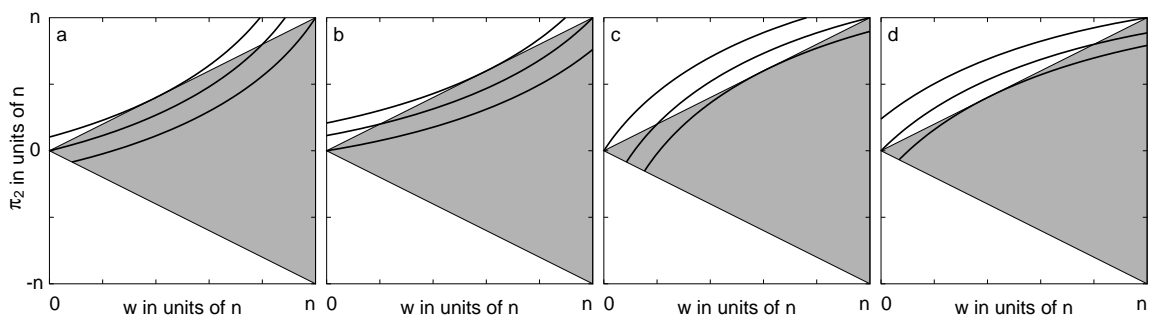

Fig. 7. Possible generic deformations of the constant level sets of $\mathcal{H}_{\text {snf }}(32)$ when $a / b \approx 1$. All cases have an extra $\mathbf{Z}_{2}$ equivalent pair of relative equilibria. In the leftmost situation (a) the double pinched torus is decomposed into a $\mathbf{Z}_{2}$ equivalent pair of single pinched tori.

This doubly pinched 2-torus is the fiber over the $(0,0)$ point in the range of the energy momentum map $\mathcal{E} \mathcal{M}$ of the integrable system $\left(\mathcal{H}_{\text {snf }}, \mathcal{H}_{1}\right)$. Thus the energy momentum map $\mathcal{E} \mathcal{M}$ has monodromy [12] when the values of $\alpha^{2}$ lie in the interval $\mathcal{I}(33)$.

\subsection{Monodromy of the generic second normal form}

The careful reader should have noticed that the reduced second normal form $\widetilde{\mathcal{H}}_{\text {snf }}^{(1)}(32)$ truncated at order one (which corresponds to second order of the first normal form $\mathcal{H}_{\mathrm{fnf}}$ ) is not generic. Indeed, when $|a / b|=1$ the level sets of $\widetilde{\mathcal{H}}_{\text {snf }}^{(1)}$ are parallel to one of the edges of $V_{n, 0}^{0}$ (and thus the corresponding level set of $\mathcal{H}_{\text {snf }}^{(1)}$ coincides with part of $P_{n, 0} \cap\left\{\pi_{3}=0\right\}$ ), see figs. 5 and 6 . In a generic situation the level sets of $\widetilde{\mathcal{H}}_{\text {snf }}$ are slightly curved.

The two possible level sets of the generic $\widetilde{\mathcal{H}}_{\text {snf }}$ are illustrated in fig. 7 . The level sets near the edge $\left|\pi_{2}\right|=w$ of $V_{n, 0}^{0}$ can either curve "inward" as in fig. 7 (a) and (b) or "outward" as in fig. 7 (c) or (d). To find which situation occurs in our problem, the fourth order of the first normal form $\mathcal{H}_{\text {fnf }}$ (which corresponds to the third order $\mathcal{H}_{\mathrm{snf}}^{(3)}$ of the second normal form) should be computed [35]. The terms $a^{\prime} \pi_{1}^{2} \pi_{2}, b^{\prime} \pi_{1}^{4}$ and $c^{\prime} \pi_{2}^{2}$ in $\mathcal{H}_{\text {snf }}^{(3)}$ ensure that the level sets of $\widetilde{\mathcal{H}}_{\text {snf }}^{(3)}$ are curved. According to our fourth-order analysis [36] both the "inward" and "outward" cases occur.

When $|a / b| \approx 1$ and $c=0$ the generic $\widetilde{\mathcal{H}}_{\text {snf }}^{(3)}$ has an extra pair of $\mathbf{Z}^{2}$-equivalent relative equilibria. These correspond to a point of tangency of a level set of $\widetilde{\mathcal{H}}_{\text {snf }}^{(3)}$ with one of the edges of $V_{n, 0}^{0}$. As $a / b$ changes the point of tangency moves quickly to one of the endpoints of the edge and disappears. There are two bifurcations involved in this process. At the first, the $\mathbf{Z}^{2}$-equivalent pair of relative equilibria appear from the singular points $\pi_{2}=0, V_{1}= \pm n$ (that is, $\pi_{2}=w=0$ ) of the second reduced phase space $P_{n, 0}$. At the second, this pair collapses to one of the $\mathbf{Z}_{2}$-symmetric relative equilibria $w=\left|\pi_{2}\right|=n$. The first 
bifurcation is a $\mathbf{S}^{1} \times \mathbf{Z}^{2}$-symmetric Hamiltonian Hopf bifurcation [36], whereas the second is a pitchfork bifurcation.

When $|a / b| \approx 1$ and $c=0$ [near the limits of the monodromy interval $\mathcal{I}(33)$ ] the topology of the level sets of the generic $\mathcal{H}_{\text {snf }}$ (which lie near $\left|\pi_{2}\right|=w$ ) can be quite complicated. In particular, fig. 7 (a) shows how the zero-level set which corresponds to the doubly pinched 2-torus in $M_{0}$, see fig. 6 , splits into two singly pinched 2-tori. Even though the topology of the zero-level set of $\widetilde{\mathcal{H}}_{\text {snf }}^{(3)}$ is different from the topology of the zero-level set of $\widetilde{\mathcal{H}}_{\text {snf }}^{(1)}$, the monodromy does not change because $\widetilde{\mathcal{H}}_{\text {snf }}^{(1)}$ and $\widetilde{\mathcal{H}}_{\text {snf }}^{(3)}$ on $M_{0}$ are smoothly homotopic and monodromy is a homotopy invariant. Consequently our geometric analysis of the nongeneric second normal form $\widetilde{\mathcal{H}}_{\text {snf }}^{(1)}$ is adequate for determining the monodromy.

\section{Quantum monodromy}

Traditionally, manifestations of monodromy in quantum systems have been analyzed using the quantum analogue of the energy momentum map [13-15]. The EBK quantization conditions for an integrable system select regular sequences of invariant tori which correspond to quantum energy levels. The global structure of energy levels of the quantum analogue of an integrable system with monodromy is quite particular and provides a very clear manifestation of monodromy [13,10,11,14]. Locally, the energy levels (and the corresponding tori) form a regular lattice of points in the range of energy momentum map $\mathcal{E} \mathcal{M}$ and can be labeled by the values of quantized actions. However, if monodromy is present, the structure of this lattice in the vicinity of the image of the pinched torus makes any global labeling impossible.

\subsection{Quantum analogue of the normal form}

The technique to construct the quantum analogue of the normalized Kepler Hamiltonian (of the first reduced Hamiltonian $\mathcal{H}_{\mathrm{fnf}}$ on $\mathrm{S}_{n / 2}^{2} \times \mathrm{S}_{n / 2}^{2}$ ) is well known, see $[37,38]$. Thus to construct the quantum analogue $\hat{\mathcal{H}}_{\text {snf }}$ of the second reduced Hamiltonian $\mathcal{H}_{\mathrm{snf}}\left(\pi_{1}^{2}, \pi_{2}\right)(32)$ we represent the latter in terms of components of the 3 -vectors $x$ and $y$ in (15) and then replace $x$ and $y$ for their quantum analogues. The Poisson algebra (17) is the algebra $\mathrm{su}(2) \times \operatorname{su}(2)$ of two angular momenta. It corresponds to the algebra of quantum angular momentum operators

$$
\left[\hat{x}_{a}, \hat{x}_{b}\right]=i \varepsilon_{a b c} \hat{x}_{c}, \quad\left[\hat{y}_{a}, \hat{y}_{b}\right]=i \varepsilon_{a b c} \hat{y}_{c}, \quad \text { and } \quad\left[\hat{x}_{a}, \hat{y}_{b}\right]=0
$$


where $\{a b c\}=\{123\}$ and $[A, B]=A B-B A$. The Casimirs of this algebra are $x^{2}$ and $y^{2}$ in (16). They are integrals of the second normal form, so that in quantum mechanics $\left[\hat{\mathcal{H}}_{\mathrm{snf}}, \hat{x}^{2}\right]=\left[\hat{\mathcal{H}}_{\mathrm{snf}}, \hat{y}^{2}\right]=0$. The standard angular momentum quantization gives

$$
\hat{x}^{2}=\hat{y}^{2}=j(j+1), \quad j=0, \frac{1}{2}, 1, \frac{3}{2}, \ldots
$$

Here $j$ labels the natural su(2) representation of dimension $2 j+1$. The $(2 j+1)^{2}$ quantum states with quantum number $j$ form an $n$ shell of the perturbed hydrogen atom system with the number of states when expressed in terms of the principal quantum number

$$
n=2\left\langle\hat{x}^{2}+\hat{y}^{2}\right\rangle=\left\langle\hat{\mathbf{T}}^{2}+\hat{\mathbf{V}}^{2}\right\rangle=1,2,3, \ldots,
$$

being $n^{2}$. Consequently,

$$
j=\frac{n-1}{2} \text {. }
$$

It follows from (16) that

$$
\hat{x}^{2}=\hat{y}^{2}=j(j+1)=\frac{n^{2}-1}{4}=\frac{\left\langle\hat{N}^{2}\right\rangle}{4}
$$

and that the classical value of the Kepler integral $N$ is

$$
n_{\mathrm{cl}}=\sqrt{\left\langle\hat{N}^{2}\right\rangle}=\sqrt{n^{2}-1}
$$

At the same time the quantum number $m$ of the integral of motion $T_{1}=x_{1}+y_{1}$ (the projection of the angular momentum $\mathbf{T}$ on the axis of the dynamical $\mathbf{S}^{1}$ symmetry) takes all integer values in the interval

$$
m=\left\langle\hat{T}_{1}\right\rangle=m_{x}+m_{y}=-2 j, \ldots, 2 j=-(n-1), \ldots, n-1 .
$$

(Here $m_{x}$ and $m_{y}$ correspond to the projection operators $\hat{x}_{1}$ and $\hat{y}_{1}$ respectively.) The classical value $c$ of $T_{1}$ equals $m$. To find the energies we solve a simple matrix problem for each value of $m$ at a fixed value of quantum number $n$. In the standard spherical harmonic basis $\left|j, m_{x}, m_{y}\right\rangle=Y_{j, m_{x}} Y_{j, m_{y}}$ with $m_{x}+m_{y}=m$, we obtain a Hermitian matrix of dimension $2 j+1-m=n-m$ which can be further reduced if the $\mathbf{Z}_{2} \times \mathbf{Z}_{2}$ symmetry is taken into account. For the Hamiltonian $\hat{\mathcal{H}}_{\text {snf }}(32)$, this matrix is tridiagonal [39]. Thus our calculation essentially reproduces [37]. 

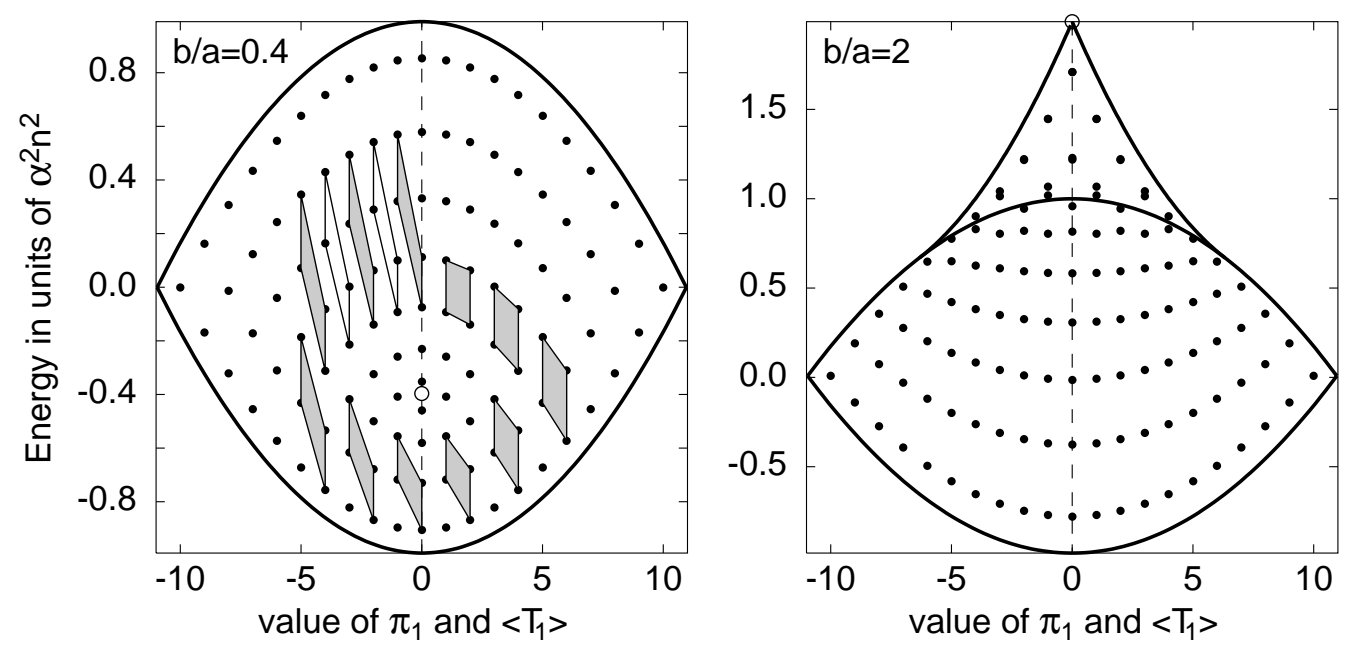

Fig. 8. Quantum and classical energy momentum map for quantum number $n=11$ and classical value $n_{\mathrm{cl}}=\sqrt{120}$. In the case with monodromy (left) the position of the pinched torus is marked by a white circle and the deformation of the local lattice is shown by a sequence of shaded quadrilaterals.

\subsection{Analysis of quantum energy momentum map}

Results of our computation for $n=11$ and the corresponding classical value of $n_{\mathrm{cl}}=\sqrt{120}$ are shown in fig. 8. Black dots in this figure show the eigenvalues of the matrix of $\hat{\mathcal{H}}_{\mathrm{snf}}$ in the basis with $n=11$ and $m=-10, \ldots, 10$, bold lines represent stationary points of $\mathcal{H}_{n, 0}$ on $P_{n, 0}$ with $n_{\mathrm{cl}}=\sqrt{120}$. These lines limit the range of the classical energy momentum map $\mathcal{E} \mathcal{M}$. The case with monodromy $\left(a / b=0.4\right.$ and $\left.\alpha^{2} \sim 0.295\right)$ is shown on the left of fig. 8. We compare and analyze quantum energy momentum map for $n=11$ and classical $\mathcal{E M}$ for $n_{\mathrm{cl}}=\sqrt{120}$.

It can be seen that quantum energies form a 2-lattice in the range of $\mathcal{E} \mathcal{M}$. In the presence of monodromy this lattice has a point defect located at the value of $\mathcal{E M}$ corresponding to the pinched torus. The type of the defect is related to the number and type of the pinch points. To visualize this defect we can define an elementary cell of the lattice and transport it along a path which lies entirely in the domain of regular values of $\mathcal{E M}$ and goes around the defect (fig. 8, left). We can easily follow the evolution of this cell because each small step to a neighboring cell is unambiguous. However, after making a tour our final cell does not match the original cell! The accumulated deformation is described by the matrix $\left(\begin{array}{ll}1 & 2 \\ 0 & 1\end{array}\right)$ where 2 corresponds to the number of pinch points of the singular fiber of the energy-momentum map [32]. Thus the lattice in fig. 8 (left) cannot be labeled globally by two quantum numbers.

For comparison, we show on the right of fig. 8 the results of the same calculation for $\alpha^{2} \sim 0.158$. In this case the value of $\alpha^{2}$ lies outside the monodromy interval 
$\mathcal{I}$ and is close to the Stark limit where $\alpha=0$. The corresponding energy level spectrum is quite similar to that in the quadratic Zeeman effect [40]. Two distinct regions in the range of $\mathcal{E} \mathcal{M}$ are clearly separated by the energy of an unstable $\mathbf{Z}_{2}$ symmetric stationary point of $\mathcal{H}_{n, c}$. In the lower region the quantum lattice corresponds to that of a rotator with angular momentum quantum number $J=n, n-1, \ldots$. The upper region corresponds to the double well 2-oscillator. Over each of the regions (except, perhaps, for a few levels near their common boundary) there is a straightforward unambiguous labeling with two quantum numbers. From (32) we can see that $b / a \geq-3 / 2$, and that to the order used in our second reduced Hamiltonian $\mathcal{H}_{n, c}$ the structure at $b / a<-1$ is qualitatively the same as in fig. 8, right, with the energy axis flipped.

The whole parametric family of $\mathcal{E} \mathcal{M}$ can be easily imagined if we note that in the Zeeman limit at $\alpha=0$ the Hamiltonian $\mathcal{H}_{n, 0}$ has an absolute minimum at the singular points $p_{1}$ and $p_{2}$ of $P_{n, 0}$. As the value of $\alpha^{2}$ increases, the value $\mathcal{H}_{n, 0}\left(p_{1}\right)=\mathcal{H}_{n, 0}\left(p_{2}\right)$ also increases. Thus the double-well region shrinks. When $\alpha^{2}=\sqrt{6} / 2-1, \mathcal{H}_{n, 0}\left(p_{1}\right)$ enters the (upper) rotator region. Here the points $p_{1}$ and $p_{2}$ become hyperbolic relative equilibria and their stable and unstable manifolds connect. After reconstruction they form a double pinched torus in $M_{0}$. In this region the angular momentum quantization rule breaks down (fig. 8, left). As $\mathcal{H}_{n, 0}\left(p_{1}\right)$ continues to increase, it becomes an absolute maximum when $\alpha^{2}>\sqrt{2} / 2$ (fig. 8 , right).

\subsection{Comparison with early quantum calculations}

Analysis of the quantum crossed fields problem goes back to 1983 when Solov'ev [28] analyzed the energy level system using an $n$-shell second order perturbation theory. He realized that the first order problem remained degenerate (indeed, for a given value of $m$ which Solov'ev calls $q=n^{\prime}+n^{\prime \prime}$, there are $n-m$ states with the same first order correction $\left.\varepsilon\left\langle T_{1}\right\rangle\right)$. He proceeded with diagonalizing his second order correction on the subspace of $n$-shell functions with fixed $m$ (sec. 3 of [28]). His resulting zeroth order equivalent operator $\Lambda_{q}$ (eq. 10 of [28]) is a direct quantum analogue of the second normal form $\mathcal{H}_{n, c}$ (32) obtained by averaging $\mathcal{H}_{\text {fnf }}$ along the orbits of $X_{T_{1}}$.

Later Solov'ev and Braun [37] calculated quantum energies for $\Lambda_{q}$ of [28] in essentially the same way as we do above. Using the field strength ratio as a parameter they distinguished three different domains of the parameter values, including the one which we call the monodromy interval $\mathcal{I}(33)$. The two relative equilibria corresponding to the singular points on $P_{n, 0}$ appeared as singularities of the effective semiclassical "potential" $U(k)$ shown in fig. 5 of [37]. When the values of the scaled field parameter $\alpha^{2}$ was contained in the interval $\mathcal{I}$, the authors associated these singular points with a "quasibarrier". 
When comparing classical and quantum results we should be well aware of the difficulty presented by the two different parameterization schemes (cf. Secs. IIE and IVB1 in [20]). In brief, we tend to use energy-scaled field strengths in classical mechanics and $n$ scaled field strengths in quantum mechanics. In other words, in the classical problem we work on the same energy level set of (1), whereas in the quantum problem we compute energies of the states within the same $n$-shell. Formally, the energy of our system can be found as follows. If $h$ is the value of $\widetilde{\mathcal{H}}_{\text {snf }}$ in (32) (plus a constant) then, taking all our rescalings properly into account,

$$
c+\frac{1}{4}(\varepsilon n) h+\cdots=\frac{2}{\varepsilon n}-\frac{2}{(\varepsilon n)^{2}} \frac{2 \varepsilon}{\omega} .
$$

The above equation can be rewritten as a formal power series in $\varepsilon n$

$$
S=(\varepsilon n)-\frac{1}{2}(\varepsilon n)^{2} c-\frac{1}{8} h(\varepsilon n)^{3} .
$$

Inverting (39b) gives

$$
\varepsilon n=S+\frac{c}{2} S^{2}+\left(\frac{c^{2}}{2}+\frac{h}{8}\right) S^{3}+\cdots .
$$

Recalling the discussion in section 3.1 we find that the energy is

$$
E=-\frac{C^{2}}{2 n^{2}}\left[1+S c+S^{2}(h+5 c) / 4+\cdots\right]
$$

In $(39 \mathrm{~d})$ the unperturbed hydrogen atom energy is factored out. The smallness parameter $S[33]$ is a uniform field strength parameter. Its $n$-shell definition can be obtained from the energy-scaled formulae in section 3.1 if the KS frequency $\omega$ is replaced by $2 / n$ so that

$$
S \rightarrow n \sqrt{\left(\frac{G n^{2}}{C^{2}}\right)^{2}+\left(\frac{3 F n^{3}}{C^{6}}\right)^{2}} .
$$

The two definitions are equivalent in the unperturbed Kepler problem. In our perturbed problem a simple replacement (40) gives the principal order terms and is sufficient for the present qualitative comparison. A more correct calculation requires the reparameterization of the first normal form $\mathcal{H}_{\text {fnf }}$ using the value of $n$ instead of $\omega$ in the definition of scaled field strengths.

Figure 9 illustrates the analysis of the energy level system carried out by authors of [37] (cf. their Fig. 4). Since the energy level structure is analyzed separ- 


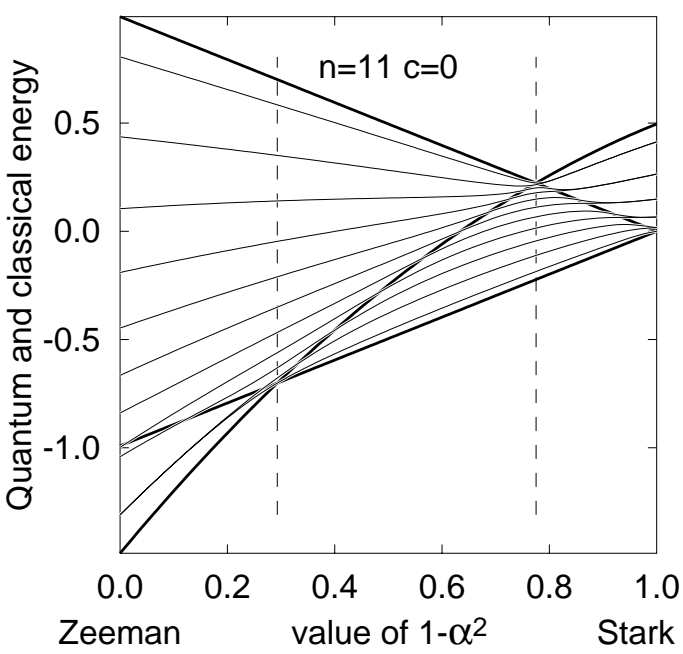

Fig. 9. Correlation diagram. Thin lines show the evolution of quantum energies (eigenvalues of the matrix of $\mathcal{H}_{n, c}$ ) with quantum number $n=11$ and $\left\langle T_{1}\right\rangle=0$ between the Zeeman and Stark limits. Bold lines represent the energy of relative equilibria of $\mathcal{H}_{n, c}$ with classical value $n_{\mathrm{cl}}=\sqrt{120}$ and $c=0$. Dashed lines mark the monodromy interval $\mathcal{I}$.

ately each $c$ (or $m$ ), monodromy cannot be understood this way. On the other hand, we can clearly observe the correspondence between the quantum spectrum and the energies of relative equilibria shown by bold lines. These latter give the limits of the quantum spectrum. In addition, they show the threshold at which doublets of levels (corresponding to the double well 2-oscillator) appear/disappear near the Stark and the Zeeman limit.

\section{Discussion}

We have demonstrated explicitly that the problem of hydrogen atom in orthogonal (crossed) magnetic and electric fields has the nontrivial property of monodromy. Our analysis develops geometric techniques which allow monodromy to be studied in other problems involving the hydrogen atom in fields. We have paid proper attention to the singularities of the second reduced phase space.

Our work raises a number of important questions. We have relied on normalization and attempted to extend the phenomenon of monodromy to systems which are nonintegrable in principle but which still have most of their KAM tori intact. Since this phenomenon is associated with the global organization of the whole family of invariant tori, we have assumed that it is stable under small perturbations and have demonstrated that as such it exists in the hydrogen atom in crossed fields. At the same time, more detailed understanding of monodromy, or rather of its analogue in such systems remains to be achieved. 
In particular, we would be greatly interested in the analysis of local actionangle variables for the Cantor sets of KAM tori surrounding the heteroclinic tangle which corresponds to the doubly pinched torus of our integrable approximation. Since these KAM tori fit together into smooth families of tori, the monodromy present in the integrable approximation survives perturbation and as such exists in the hydrogen atom problem in orthogonal electric and magnetic fields when the parameter $\alpha$ lies in the interval $\mathcal{I}$.

A different group of questions is associated with "quantum monodromy." Here again one should attempt to generalize our methods to quantum systems whose classical analogues are not integrable, but which can be treated within the framework of quantum perturbation theory. When applying the ideas of this paper to quantum systems, one should be aware of differences between the classical and quantum normal form algorithms [41].

Persistence of quantum monodromy under small perturbations is also a subject of study on its own. We are, nevertheless, convinced that future studies of the hydrogen atom in crossed fields will reveal the energy level structure which we obtained for the quantized integrable approximation and which is characteristic to all systems with monodromy. Such studies can answer a very interesting question of how far this structure will persist with increasing perturbation (energy).

Furthermore, perturbed hydrogen atom and the crossed field system in particular $[42,43]$ continue to attract considerable interest of experimentalists. Application of the idea of monodromy in experimental studies depends on how the above questions are answered. We think that our system will become experimentally important precisely because it can, ideally, be "tuned" in and out of the interval $\mathcal{I}$ of field parameter $\alpha$ where monodromy exists.

\section{Acknowledgement}

This work has been initiated and encouraged at the workshops organized by Dr. Mark Roberts at the Mathematics Research Centre, University of Warwick, UK, in March 1997 and 1999. The idea of our discussion of quantum monodromy in terms of point defects and elementary cells of 2-lattices (sec. 5) is mainly due to Prof. Boris Zhilinskii [11] whom we further thank for many discussions. We also thank Prof. John Delos for his improvement of fig. 8 . 


\section{References}

[1] J. J. Duistermaat, Communs. Pure Appl. Math. 33, 687 (1980).

[2] R. H. Cushman and L. M. Bates, Global Aspects of Classical Integrable Systems (Birkhäuser Verlag, Basel, 1977); see Appendix D.

[3] R. Cushman, Centrum voor Wiskunde en Informatica Newsletter 1, 4 (1983); see Chap. IV of Ref. [2]

[4] R. Cushman and J. C. van der Meer, Lect. Notes Math. 1416, 26 (1991).

[5] R. Cushman and H. Knörrer, Lect. Notes Math. 1139, 12 (1985).

[6] J. C. van der Meer, The Hamiltonian Hopf Bifurcation, Lect. Notes Math. 1160 $(1985)$.

[7] L. Bates, Z. Angew. Math. Phys. 6, 837, (1991).

[8] M. Zou, J. Geom. Phys. 10, 37 (1992).

[9] L. Bates and R. Cushman, Meccanica 30, 271 (1995).

[10] For a brief account of the present analysis see R. H. Cushman and D. A. Sadovskií, Europhys. Lett. (1999), in press.

[11] D. A. Sadovskií and B. I. Zhhilinskií, Physics. Lett. (1999), in press.

[12] R. Cushman and J. J. Duistermaat, Non-Hamiltonian monodromy, preprint, (Utrecht, 1997).

[13] R. Cushman and J. J. Duistermaat, Bull. Am. Math. Soc. 19, 475 (1988).

[14] Vĩ Ngoc San, Quantum monodromy in integrable systems, preprint 1055 (University of Utrecht, 1998);

[15] M. S. Child, J. Phys. A 31, 657 (1998); M. S. Child, T. Weston, and J. Tennyson, submitted to Mol. Phys. (1998).

[16] M. Kuwata, A. Harada, and H. Hasegawa, J. Phys. A: Math. Gen. 23, 3227 (1990).

[17] D. Farrelly, J. Chem. Phys. 85, 2119 (1986); D. Farrelly and K. Krantzman, Phys. Rev. A 43, 1666 (1991); K. D. Krantzman, J. Milligan, and D. Farrelly, Phys. Rev. A 45, 3093 (1992); D. Farrelly and J. A. Milligan, Phys. Rev. A 45, 8277 (1992).

[18] D. Farrelly, T. Uzer, P. E. Raines, J. P. Skelton, and J. Milligan, Phys. Rev. A 45, 4738 (1992).

[19] D. A. Sadovskií, B. I. Zhilinskií, and L. Michel, Phys. Rev. A 53, 4064 (1996).

[20] D. A. Sadovskií and B. I. Ẑhilinskií, Phys. Rev. A 57, 2867 (1998). 
[21] J. C. van der Meer and R. H. Cushman, in Proc. of the XV Intl. Conference on Diffrential Geometric Methods in Theoretical Physics, edited by H. Doebner and J. Henning (World Scientific, Singapore, 1987), p. 403.

[22] R. H. Cushman, in Dynamics Reported, new series, vol. 1, edited by C. K. Jones et al (Springer, New York, 1992) p. 54.

[23] D. A. Sadovskií, unpublished.

[24] T. Uzer, Phys. Rev. A 42, 5787 (1990); A. R. P. Rau and L. Zhang, Phys. Rev. A 42, 6342 (1990); J. P. Salas, A. Deprit, S. Ferrer, V. Lanchares, and J. Palacián, Phys. Lett. A 242, 83 (1998).

[25] M. J. Gourlay, T. Uzer, and D. Farrelly, Phys. Rev. A 47, 3113 (1993); J. von Milczewski, G. H. F. Diercksen, and T. Uzer, Phys. Rev. Lett. 73, 2428 (1994); Intl. J. Bif. Chaos, 4905 (1994).

[26] E. Flöthmann, J. Main, and K. W. Welge, J. Phys. B 27, 2821 (1994); E. Flöthmann and K. W. Welge, Phys. Rev. A 54, 1884 (1996).

[27] J. von Milczewski, D. Farrelly, and T. Uzer, Phys. Rev. Lett. 78, 2349 (1997).

[28] Solov'ev E. A., Sov. Phys. JETP 58, 63 (1983). In the notation of Solov'ev $q$ equals our $c$ or $m, A$ corresponds to vector $K$, and $I_{1}$ and $I_{2}$ to $x$ and $y$.

[29] We ignore all effects due to the spin of the electron, relativistic corrections, and we also simplify the two-body problem by considering an infinitely heavy nucleus (while in reality $m_{p} / m_{e} \approx 1836$ ).

[30] As in [20] and elsewhere we use atomic units.

[31] See [2], notes on p. 408.

[32] Nguyen Tien Zung, Diff. Geom. Appl. 7, 123 (1997).

[33] In the notation of [20] $\alpha=G_{s}, \beta=F_{s}$ and $\varepsilon=\frac{\tau}{n}=S \frac{\omega}{2}$, where $S=$ $C\left(\frac{2}{C \omega}\right)^{2} \sqrt{G^{2}+\left(3 F \frac{2}{C \omega}\right)^{2}}$ is the scaled uniform field intensity used as a smallness parameter. In [20] the symmetry group $\mathrm{Z}_{2} \times \mathrm{Z}_{2}$ with operations $\left(e, \sigma_{1}, \sigma_{2}, \sigma_{3}\right)$ is called $G_{4}$ with operations $\left(I, T_{2}, \sigma, T_{s}\right)$.

[34] Often called after Laplace-Runge-Lenz, see [2], p. 400, note to p. 55.

[35] It can be shown using invariant theory [23] that the ring of all polynomials in $\left(T_{1}, T_{2}, T_{3}, V_{1}, V_{2}, V_{3}\right)$ which are invariant with respect to the $\mathbf{S}^{1} \times \mathbf{Z}_{2} \times \mathbf{Z}_{2}$ action on $\mathrm{S}_{n / 2}^{2} \times \mathrm{S}_{n / 2}^{2}$ is freely generated by $\left(\pi_{2}, V_{1}^{2}, T_{1}\right)$. Polynomials in the $k$-th order of the second normal form $\mathcal{H}_{\text {snf }}$ are of degree $k+1$ in $T$ 's and $V$ 's. Consequently, 
[cf. (24)], to third order $\mathcal{H}_{\mathrm{snf}}$ has the following terms

\begin{tabular}{cl} 
order of $\mathcal{H}_{\mathrm{snf}}$ & invariant polynomials \\
\hline 1 & $T_{1}$ \\
$\varepsilon$ & $\pi_{2}, V_{1}^{2}, T_{1}^{2}$ \\
$\varepsilon^{2}$ & $\pi_{2} T_{1}, V_{1}^{2} T_{1}, T_{1}^{3}$ \\
$\varepsilon^{3}$ & $\pi_{2} T_{1}^{2}, V_{1}^{2} T_{1}^{2}, T_{1}^{4}, \pi_{2}^{2}, \pi_{2} V_{1}^{2},\left(V_{1}^{2}\right)^{2}$
\end{tabular}

[36] R. H. Cushman and D. A. Sadovskií, Degenerate Hamiltonian Hopf bifurcation, in preparation.

[37] Braun P. A. and Solov'ev E. A., Sov. Phys. JETP 59, 38 (1984). These authors use $b \approx \alpha^{-2}-2 \alpha^{2}-2$ as a field parameter.

[38] Robnik M., J. Phys. A 17, 109 (1984). Robnik M. and Schrüfer E., J. Phys. A 18, L853 (1985).

[39] Using creation-annihilation operators $\hat{x}^{ \pm}=\hat{x}_{2} \pm i \hat{x}_{3}$ and $\hat{y}^{ \pm}=\hat{y}_{2} \pm i \hat{y}_{3}$ the Hamiltonian $\hat{\mathcal{H}}_{\text {snf }}$ can be put in terms of the operator $\hat{x}^{+} \hat{y}^{-}+\hat{x}^{-} \hat{y}^{+}$which contributes to the second diagonal and terms $\hat{x}_{1}^{2}, \hat{y}_{1}^{2}$, and $\hat{x}_{1} \hat{y}_{1}$ which contribute to the main diagonal. Of course, all these operators preserve $m_{x}+m_{y}=c$.

[40] Solov'ev E. A., JETP Lett. 34, 265 (1981); Sov. Phys. JETP 55, 1017 (1982). Herrick D. R., Phys. Rev. A 26, 323 (1982).

[41] M. Kummer, Fields Inst. Communs. 8, 35 (1996).

[42] G. Wiebusch, J. Main, K. Kruger, H. Rottke, A. Holle, and K. Welge, Phys. Rev. Lett. 62, 2821 (1989); G. Reithel and H. Walther, Phys. Rev. A 49, 1646 (1993).

[43] C. Neumann, R. Ubert, S. Freund, E. Flöthmann, B. Sheehy, K. H. Welge, M. R. Haggerty, and J. B. Delos, Phys. Rev. Lett. 78, 4705 (1997). 